\title{
Hear the Sound of Weyl Fermions
}

\author{
Zhida Song ${ }^{1,2}$ and Xi Dai ${ }^{1, *}$ \\ ${ }^{1}$ Department of Physics, Hong Kong University of Science and Technology, \\ Clear Water Bay, Kowloon, Hong Kong \\ ${ }^{2}$ Department of Physics, Princeton University, Princeton, New Jersey 08544, USA
}

(Received 4 February 2019; revised manuscript received 10 April 2019; published 17 June 2019)

\begin{abstract}
Quasiparticles and collective modes are two fundamental aspects that characterize quantum matter in addition to its ground-state features. For example, the low-energy physics for Fermi-liquid phase in He-III is featured not only by fermionic quasiparticles near the chemical potential but also by fruitful collective modes in the long-wave limit, including several different sound waves that can propagate through it under different circumstances. On the other hand, it is very difficult for sound waves to be carried by electron liquid in ordinary metals due to the fact that long-range Coulomb interaction among electrons will generate a plasmon gap for ordinary electron density oscillation and thus prohibits the propagation of sound waves through it. In the present paper, we propose a unique type of acoustic collective mode in Weyl semimetals under magnetic field called chiral zero sound. Chiral zero sound can be stabilized under the so-called "chiral limit," where the intravalley scattering time is much shorter than the intervalley one and propagates only along an external magnetic field for Weyl semimetals with multiple pairs of Weyl points. The sound velocity of chiral zero sound is proportional to the field strength in the weak field limit, whereas it oscillates dramatically in the strong field limit, generating an entirely new mechanism for quantum oscillations through the dynamics of neutral bosonic excitation, which may manifest itself in the thermal conductivity measurements under magnetic field.
\end{abstract}

DOI: 10.1103/PhysRevX.9.021053

Subject Areas: Condensed Matter Physics

\section{INTRODUCTION}

Topological semimetals are unique metallic systems with a vanishing density of states at the Fermi level [1-11]. Among different topological semimetals, the Weyl semimetal [2-5] is the most robust one because it requires no particular crystalline symmetry to protect it. The low-energy quasiparticle structure of a Weyl semimetal usually contains several pairs of Weyl points (WPs), isolated crossing points in 3D momentum space formed by energy bands without degeneracy. Near each WP, the surrounding quasiparticles can be well described by the Weyl equation proposed by Weyl 90 years ago in the context of particle physics [12]. The WP provides not only the linear energy dispersion around it, but more importantly, the "monopole" structure in the Berry curvature, which makes the dynamics of these Weyl quasiparticles completely different from free electrons in ordinary metals or semiconductors and leads to many exotic properties of the Weyl semimetal, i.e., the Fermi-arc behavior [5,13-18]

\section{daix@ust.hk}

Published by the American Physical Society under the terms of the Creative Commons Attribution 4.0 International license. Further distribution of this work must maintain attribution to the author(s) and the published article's title, journal citation, and DOI. on the surface and the negative magnetoresistance [19-23] caused by the chiral anomaly [1,24-27].

So far, the Weyl semimetal is considered a new topological state in condensed matter physics only because of its unique quasiparticle dynamics, which manifests itself in various transport experiments [19-23]. On the other hand, the unique collective modes are other types of features that characterize a new state of matter which is yet to be revealed for Weyl semimetal systems [18,28-36]. The most common collective mode in a liquid system is sound, which usually requires collisions to propagate. For a neutral Fermi liquid such as He-III [37-40], ordinary sound can exist only when $\omega \tau \ll 1$, where $\tau$ is the lifetime of the quasiparticles. For a clean system, the low-energy quasiparticle lifetime approaches infinity with reducing temperature, which prohibits the existence of normal sound modes at low enough temperature when $\omega \tau \gg 1$. However, there is a completely different type of sound that emerges in the above "collisionless region" called zero sound, which is purely generated by the quantum-mechanical many-body dynamics under the clean limit [37,41-44]. In a typical Fermi-liquid system, zero sound can be simply viewed as the deformation of the Fermi surface that oscillates and propagates in the system with the "restoration force" provided by the residual interaction among the quasiparticles around the Fermi surface. Like other types of elementary excitations in 
condensed matter, the form functions of zero sound modes carry irreducible representations (irreps) of the symmetry group of the particular system. For an electron liquid in a normal metal, the density oscillation corresponding to the trivial representation is always governed by the long-range Coulomb interaction and becomes the well-known plasmon excitation with a finite gap in the long-wave limit. Thus, zero sound modes can exist only in high multipolar channels ascribing to the nontrivial representation of the symmetry group, within which the residual interactions among the quasiparticles are positive definite. The above condition requires a strong and anisotropic residual interaction in solids, which is difficult to be realized in normal metals.

One of the exotic phenomena of a Weyl semimetal is the chiral magnetic effect (CME) [24,45-48], where each valley will contribute a charge current under the external magnetic field. The "anomalous current" contributed by the CME from a single WP valley with positive (negative) chirality is always parallel (antiparallel) to the field direction with its amplitude being proportional to the particle number of that particular valley. To be specific, the anomalous current contributed by the $\nu$ th valley through CME is $\mathbf{j}_{\nu}^{a}=e \mathbf{B} \chi_{\nu} /$ $\left(4 \pi^{2}\right)\left(\mu_{\nu}-\mu\right)$, where $\chi_{u}$ and $\mu_{\nu}$ denote the chirality and the imbalanced chemical potential of the $\nu$ th WP, respectively, $\mu$ is the chemical potential at equilibrium, $e=\mp|e|$ is the charge of the electronlike (holelike) quasiparticle, and $\mathbf{B}$ is the magnetic field. The above CME immediately causes an interesting consequence: The particle number imbalance among different valleys will induce particle transport and thus make it possible to form a coherent oscillation of the valley particle numbers over space and time, which is a completely new type of collective mode induced by the CME.

On the other hand, the most common collective modes in a charged Fermi-liquid system are plasmons, and for a Weyl semimetal under a magnetic field, they are such collective modes where the oscillations of the valley particle numbers cannot cancel each other and generate net-charge-density oscillation in real space [28-36]. Since these modes are coupled to the CME current, the plasmon frequencies significantly depend on the magnetic field [36]. Following Ref. [36], in this paper we call them "chiral plasmons" (CPs). In general, each of the CP modes form a trivial (identity) irrep of the symmetry group. As we discuss in detail below, among all the CPs, there are only two branches that are fully gapped (with opposite frequencies), whereas the other branches are gapless. For the simplest Weyl semimetal with only a single pair of WPs, the little group at finite wave vector $\mathbf{q}$ contains only an identity operator under magnetic filed, indicating that all the electronic collective modes propagating with wave vector $\mathbf{q}$ will generally cause net-charge-density oscillation and thus belong to different branches of the $\mathrm{CP}$ modes.

The situation becomes completely different for a Weyl semimetal with multiple pairs of WPs. Now we can have collective "breathing modes" of Fermi surfaces in different WP valleys so that they oscillate in an antiphase way and cancel out the net charge oscillation exactly, as illustrated schematically in Fig. 1(d) for two pairs of WPs. Since these antiphase modes do not cause any net charge current, the collective oscillations of the valley charge and valley current will be completely decoupled from the plasmon modes, and their dispersion relation remains gapless and linear in the long-wave limit, which is called "chiral zero sound" (CZS) in this paper. As we discuss in more detail below, the CZS modes carry the nontrivial irreps of the corresponding little group, with which we can figure out how many CZS modes can exist with the magnetic field being applied in some particular crystal directions.

In order to clearly describe the physical process in Weyl semimetal systems, we divide the charge current contributed by the $\nu$ th WP valley $\mathbf{j}_{\nu}$ into two parts: the "anomalous current" $\mathbf{j}_{\nu}^{a}$ caused by the change of the valley particle number through the CME and the "normal current" $\mathbf{j}_{\nu}^{n}$ caused by the deformation of the Fermi surface in the $\nu$ th valley. For the general situation, the two types of currents are coupled together and contribute jointly to both the $\mathrm{CP}$ and $\mathrm{CME}$ modes. However, in the present paper, we consider a specific limit where only the anomalous current can survive, and both the CP and CZS are purely contributed by the CME. Such a limit was proposed previously by Son and Yamamoto [24] requiring the intravalley relaxation time to be much shorter than the intervalley one, which guarantees that the intravalley relaxation process is fast enough so that any deformation of the Fermi surface from its equilibrium shape can be neglected. In the following, we call this limit the "chiral limit" and mainly discuss the physics of the CZS under it.

\section{BOLTZMANN'S EQUATION METHOD}

Let us first introduce the Boltzmann equation in the chiral limit. The Boltzmann method is valid only in the semiclassical limit, where $\omega_{B} \tau \ll 1, \omega_{B} \ll \mu$, and $\mu \tau \gg 1$. Here, $\omega_{B}=v_{F} \sqrt{e B}$ is the magnetic frequency, $v_{F}$ is the Fermi velocity, and $\tau$ is the quasiparticle lifetime. (In this paper, we set $\hbar=1$ and the energy of WP as 0 .) In the semiclassical limit, the level smearing caused by the finite quasiparticle lifetime is much larger than the Landau-level splitting, but it is much smaller than the chemical potential; hence, the Landau-level quantization can be ignored, and the Fermi surface remains well defined. Therefore, in the semiclassical limit, the collective dynamics of a Fermiliquid system can be described by the quasiparticle distribution function $n_{\nu}(\mathbf{k}, \mathbf{r}, t)$ through the following Boltzmann equation, where $\nu$ is the valley index, $\mathbf{k}$ is the momentum, and $\mathbf{r}$ is the position of the quasiparticle,

$$
\begin{aligned}
\frac{d \delta n_{\nu}(\mathbf{k}, \mathbf{r}, t)}{d t}= & \left(-\dot{\mathbf{r}}_{\nu} \cdot \partial_{\mathbf{r}}-\dot{\mathbf{k}}_{\nu} \cdot \partial_{\mathbf{k}}\right) \delta n_{\nu}(\mathbf{k}, \mathbf{r}, t) \\
& +S\left[\delta n_{\nu}(\mathbf{k}, \mathbf{r}, t)\right],
\end{aligned}
$$

where the first term and second term on the rhs describe the drifting motion and the scattering process, respectively. 


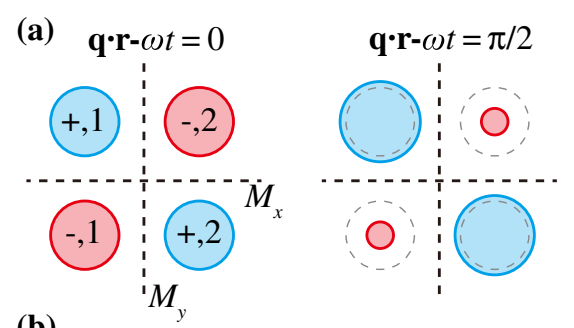

(b)
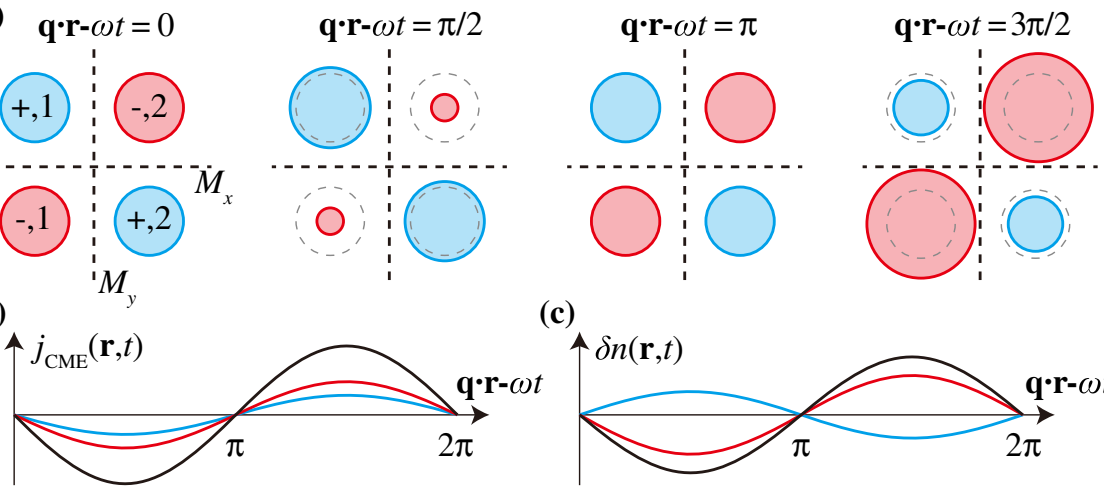

(c)

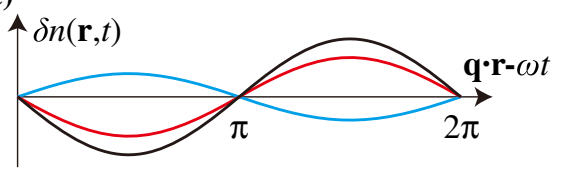

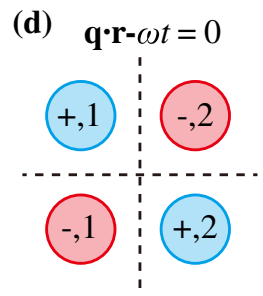

(e)
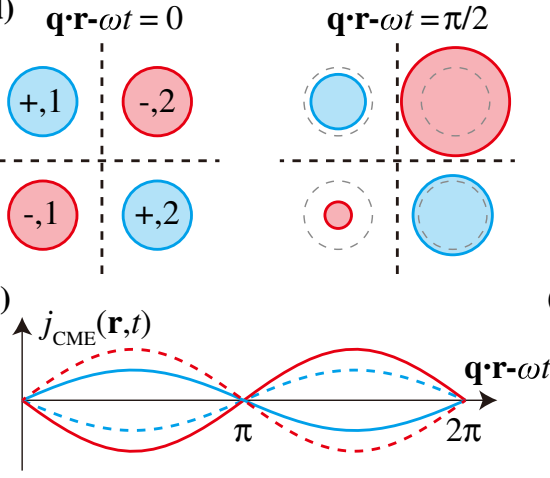
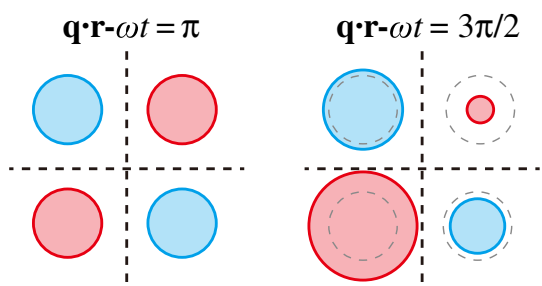

(f)

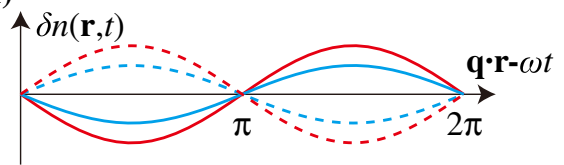

FIG. 1. Chiral zero sound and chiral plasmon modes in the minimal model with four Weyl points. The symmetry group of the model is $C_{2 v}$ consisting of a twofold rotation axis in the $z$ direction and two mirror planes in the $x-z$ plane and $y-z$ plane, respectively. In (a) and (d), dashed lines represent the two mirrors, the colored disks represent the Fermi surfaces around the WPs, and the dashed gray circles represent the Fermi surfaces in equilibrium. Here, the four WPs are labeled by $(s, a)$, with $s= \pm$ the chirality and $a=1,2$ the subvalley index. (a) The volume of Fermi surfaces as functions of space and time in the chiral plasmon mode, where $(s, 1)$ and $(s, 2)$ are always in phase, making the mode even under $C_{2}$ rotation. (b),(c) The chiral magnetic currents and quasiparticle densities as functions of space and time in the chiral plasmon mode. Here, the red and blue lines represent the contributions from the $(-, 1)[(-, 2)]$ and $(+, 1)[(+, 2)]$ Fermi surfaces, respectively, and the black lines represent the net current and density. (d) The volume of Fermi surfaces in the chiral zero sound mode, where $(s, 1)$ and $(s, 2)$ are always out of phase, making the mode odd under $C_{2}$ rotation. (e),(f) The chiral magnetic currents and quasiparticle densities as functions of space and time in the chiral zero sound mode. The contributions from the $(-, 1),(+, 1)$, $(-, 2)$, and $(+, 2)$ Fermi surfaces are represented by the red solid, blue solid, red dashed, and blue dashed lines, respectively. In the chiral zero sound mode, both the net current and the net density vanish.

(An explicit derivation of this equation is given in Appendix A). The time derivatives $\dot{\mathbf{r}}_{\nu}$ and $\dot{\mathbf{k}}_{\nu}$ are given by the equations of motion of the quasiparticles. In the presence of external field and Berry's curvature $\boldsymbol{\Omega}_{\nu}(\mathbf{k})$, they can be written as $[49,50]$

$$
\begin{aligned}
& \gamma_{\nu}(\mathbf{k}) \dot{\mathbf{k}}_{\nu}=-\partial_{\mathbf{r}} \epsilon_{\nu}+e \mathbf{v}_{\nu}(\mathbf{k}) \times \mathbf{B}+e\left(\partial_{\mathbf{r}} \epsilon_{\nu} \cdot \mathbf{B}\right) \boldsymbol{\Omega}(\mathbf{k}), \\
& \gamma_{\nu}(\mathbf{k}) \dot{\mathbf{r}}_{\nu}=\mathbf{v}_{\nu}(\mathbf{k})+\partial_{\mathbf{r}} \epsilon_{\nu} \times \boldsymbol{\Omega}_{\nu}(\mathbf{k})-e\left[\boldsymbol{\Omega}_{\nu}(\mathbf{k}) \cdot \mathbf{v}_{\nu}(\mathbf{k})\right] \mathbf{B},
\end{aligned}
$$

where $\epsilon_{\nu}(\mathbf{k})$ is the quasiparticle energy, $\mathbf{v}_{\nu}(\mathbf{k})=\partial_{\mathbf{k}} \epsilon_{\nu}(\mathbf{k})$ is the quasiparticle velocity, and $\gamma_{\nu}(\mathbf{k}, \mathbf{B})=1-e \mathbf{B}$. $\boldsymbol{\Omega}_{\nu}(\mathbf{k})$ is the phase-space volume correction due to the presence of the Berry curvature [51]. We emphasize that $\epsilon_{\nu}(\mathbf{k})$ is not the bare band energy but the renormalized quasiparticle energy due to the presence of the collective mode. To obtain $\epsilon_{\nu}(\mathbf{k})$, we first write the total energy in terms of $n_{\nu}(\mathbf{k}, \mathbf{r}, t)$,

$$
\begin{aligned}
E_{\text {total }}(t)= & E_{\text {total }}^{0}+\sum_{\nu} \int d^{3} \mathbf{r} \int \frac{d^{3} \mathbf{k}}{(2 \pi)^{3}} \gamma_{\nu}(\mathbf{k}, \mathbf{B}) \epsilon_{\nu}^{0}(\mathbf{k}) \delta n_{\nu}(\mathbf{k}, \mathbf{r}, t)+\iint d^{3} \mathbf{r} d^{3} \mathbf{r}^{\prime} \frac{e^{2}}{\epsilon_{0}\left|\mathbf{r}-\mathbf{r}^{\prime}\right|} \delta n(\mathbf{r}, t) \delta n\left(\mathbf{r}^{\prime}, t\right) \\
& +\sum_{\nu \nu^{\prime}} \int d^{3} \mathbf{r} \int \frac{d^{3} \mathbf{k}}{(2 \pi)^{3}} \gamma_{\nu}(\mathbf{k}, \mathbf{B}) \int \frac{d^{3} k^{\prime}}{(2 \pi)^{3}} \gamma_{\nu^{\prime}}\left(\mathbf{k}^{\prime}, \mathbf{B}\right) f_{\nu, \nu^{\prime}} \delta n_{\nu}(\mathbf{k}, \mathbf{r}, t) \delta n_{\nu^{\prime}}\left(\mathbf{k}^{\prime}, \mathbf{r}, t\right),
\end{aligned}
$$


where the second and third terms are the long-range Coulomb interaction and the residual short-range interaction between the quasiparticles, respectively [52]. Here, $\epsilon_{\nu}^{0}(\mathbf{k})$ is the bare energy dispersion for the quasiparticle, $\delta n_{\nu}(\mathbf{k}, \mathbf{r}, t)=n(\mathbf{k}, \mathbf{r}, t)-n_{F}\left[\epsilon_{\nu}^{0}(\mathbf{k})-\mu\right]$ is the deviation from Fermi-Dirac distribution, and $\delta n(\mathbf{r}, t)=$ $\sum_{\nu} \int\left[\left(d^{3} \mathbf{k}\right) /(2 \pi)^{3}\right] \gamma_{\nu}(\mathbf{k}, \mathbf{B}) n_{\nu}(\mathbf{k}, \mathbf{r}, t)$ is the net charge density at position $\mathbf{r}$. In general, the short-range interaction matrix $f_{\nu, \nu^{\prime}}$ in the above equation should have the full momentum dependence and be written as $f_{\nu, \nu^{\prime}}\left(\mathbf{k}, \mathbf{k}^{\prime}\right)$ [52]. However, here we consider the case where the Fermi surfaces are small enough such that the $\mathbf{k}$ dependence in $f_{\nu, \nu^{\prime}}\left(\mathbf{k}, \mathbf{k}^{\prime}\right)$ can be omitted. Then, the renormalized quasiparticle energy is given by the functional derivative of the total energy as $\epsilon_{\nu}(\mathbf{k}, \mathbf{r}, t)=\delta E_{\text {total }} / \delta n_{\nu}(\mathbf{k}, \mathbf{r}, t)$. An elaborate study of collective modes in Weyl systems with only one pair of WPs using the Boltzmann equation can be found in Ref. [31].

\section{THE CHIRAL LIMIT}

To introduce the chiral limit, we decompose $\delta n(\mathbf{k}, \mathbf{r}, t)$ into two parts: the part that keeps the quasiparticle number in each valley unchanged $\widetilde{\delta n_{\nu}}(\mathbf{k}, \mathbf{r}, t)$ and the part that changes the valley quasiparticle numbers $\overline{\delta n}_{\nu}(\mathbf{k}, \mathbf{r}, t)$. In the following, we refer to $\widetilde{\delta n}_{\nu}(\mathbf{k}, \mathbf{r}, t)$ as the Fermi-surface degrees of freedom (d.o.f.) and $\overline{\delta n}_{\nu}(\mathbf{k}, \mathbf{r}, t)$ as the valley d.o.f. Since the intravalley scattering preserves the quasiparticle number in each valley, $\overline{\delta n}_{\nu}(\mathbf{k}, \mathbf{r}, t)$ can be relaxed only through the intervalley scattering. On the other hand, $\widetilde{\delta n}_{\nu}(\mathbf{k}, \mathbf{r}, t)$ can be relaxed through both the inter- and intravalley scattering processes. Therefore, the relaxation time of $\overline{\delta n}_{\nu}(\mathbf{k}, \mathbf{r}, t)$ is always longer than the relation time of $\widetilde{\delta n_{\nu}}(\mathbf{k}, \mathbf{r}, t)$. We can approximate the scattering term as

$$
\mathcal{S}\left[\delta n_{\nu}(\mathbf{r}, \mathbf{k}, t)\right]=-\frac{\widetilde{\delta n_{\nu}}(\mathbf{r}, \mathbf{k}, t)}{\tau_{0}}-\frac{\left.{\overline{\delta n_{\nu}}}_{\nu} \mathbf{r}, \mathbf{k}, t\right)}{\tau_{v}} .
$$

As we prove in Appendix E, for the simplest case where both the inter- and intravalley scattering cross sections are constants (without $\mathbf{k}$ dependence), Eq. (5) is almost exact, and the valley d.o.f. have the form $\overline{\delta n}_{\nu}(\mathbf{k}, \mathbf{r}, t) \propto$ $\delta n_{\nu}(\mathbf{r}, t) \delta\left[\epsilon_{\nu}^{0}(\mathbf{k})-\mu\right]$. Such a k-independent scattering cross section is a good approximation for a small Fermi surface. Now we argue that in the chiral limit where $\tau_{0} \ll \tau_{v}$, the Fermi-surface degrees and the valley degrees are decoupled, and the collective modes are purely contributed by the valley degrees. To zeroth order of $\tau_{0}$, nonzero $\widetilde{\delta n_{\nu}}(\mathbf{k}, \mathbf{r}, t)$ will be relaxed to zero in an infinitely short time; hence, the Fermi-surface degrees are always in equilibrium, i.e., $\widetilde{\delta n_{\nu}}(\mathbf{k}, \mathbf{r}, t)=0$. Therefore, to obtain the dynamic equation in the chiral limit, we can simply assume $\delta n_{\nu}(\mathbf{k}, \mathbf{r}, t)=\overline{\delta n}_{\nu}(\mathbf{k}, \mathbf{r}, t)$. Here we take the trial solution as $\overline{\delta n}_{\nu}(\mathbf{k}, \mathbf{r}, t)=c_{\nu} e^{i \mathbf{q}(\cdot \mathbf{r}-\omega t)} \delta\left[\epsilon_{\nu}^{0}(\mathbf{k})-\mu\right]$, where $\mathbf{q}$ and $\omega$ are the wave vector and frequency of the corresponding collective mode, respectively. By substituting this trial solution and Eqs. (2) and (3) into Eq. (1), we obtain the following dynamic equation

$$
\begin{aligned}
\left(\omega+\frac{i}{\tau_{v}}\right) \eta_{\nu}= & \frac{e(\mathbf{q} \cdot \mathbf{B}) \chi_{\nu}}{4 \pi^{2} \beta_{\nu}(\mathbf{B})} \eta_{\nu} \\
& +\frac{e(\mathbf{q} \cdot \mathbf{B}) \chi_{\nu}}{4 \pi^{2}} \sum_{\nu^{\prime}}\left(f_{\nu, \nu^{\prime}}+\frac{e^{2}}{\epsilon_{0} \mathbf{q}^{2}}\right) \eta_{\nu^{\prime}},
\end{aligned}
$$

where $\beta_{\nu}(\mathbf{B})$ is the bare compressibility of the $\nu$ th valley, $\chi_{\nu}= \pm 1$ is the chirality, and $\eta_{\nu}=\beta_{\nu}(\mathbf{B}) c_{\nu}$ is the imbalanced quasiparticle particle number (per unit volume) for the $\nu$ th valley. At zero temperature, the bare compressibility is nothing but the density of states at the Fermi level. In the semiclassical region, there are $\beta_{\nu}(0) \sim \mu^{2} / v_{F}^{3}$ and $\beta_{\nu}(\mathbf{B})-\beta_{\nu}(0) \sim \omega_{B}^{2} / v_{F}^{3}$. In the semiclassical limit $\omega_{B} \ll \mu$, we have $\beta_{\mu}(B) \approx \beta_{\mu}(0)$.

Equation (6) is the key equation of this paper, which directly leads to both $\mathrm{CP}$ and CZS solutions. We put the rigorous derivation in Appendix B and give only a brief introduction here in the main text. We can interpret Eq. (6) as the continuity equation for the quasiparticle number in the $\nu$ th valley under the chiral limit, i.e., $\partial_{t} \eta_{\nu}+\nabla \cdot \mathbf{j}_{\nu}^{a}=0$, where $\mathbf{j}_{\nu}^{a}$ is the CME current contributed by the $\nu$ th valley. For simplicity, here we set $\tau_{v}=\infty . i \partial_{t} \eta_{\nu}$ gives the lhs and $-i \nabla \cdot \mathbf{j}_{\nu}^{a}$ gives the rhs of Eq. (6). In the chiral limit, each of the Weyl valleys can be described by the Fermi-Dirac distribution functions with time- and valley-dependent chemical potential $\mu_{\nu}$. Then, the CME current for the $\nu$ th valley $\mathbf{j}_{\nu}^{a}$ can be simply written as $\mathbf{j}_{\nu}^{a}=e \mathbf{B} \chi_{\nu} /$ $\left(4 \pi^{2}\right)\left(\mu_{\nu}-\mu\right)$, where $\mu$ is the chemical potential in equilibrium. The above anomalous current $\mathbf{j}_{\nu}^{a}$ is contributed by two effects: the change of quasiparticle number and the modification of the averaged quasiparticle energy in the $\nu$ th valley due to the interaction, which correspond to the two terms on the rhs of Eq. (6), respectively.

In the above analysis, for simplicity, we always neglect the $\mathbf{k}$ dependence in the form of residual interaction among the quasiparticles, which is a good approximation as long as all the FSs in such Weyl semimetal systems are small enough. To generalize our discussion, in Appendix $F$ we prove that even if we keep $\mathbf{k}$ dependent, the valley d.o.f. $\overline{\delta n}_{\nu}(\mathbf{k})$ are still well defined and free of intravalley scattering, but their form will be modified. Furthermore, under the chiral limit, the dynamic equation is still given by Eq. (6), except that $f_{\nu, \nu^{\prime}}$ has to be understood as the "k-averaged" interaction obtained from $f_{\nu, \nu^{\prime}}\left(\mathbf{k}, \mathbf{k}^{\prime}\right)$. Please see Appendix F for more details.

To understand more about the chiral limit, we need to find the upper bound of $\tau_{0}$ below which the zeroth-order discussion is valid. In Appendix E, we deal with the effect of finite $\tau_{0}$ in the standard second-order perturbation theory. Here we describe only the main conclusion: Finite $\tau_{0}$ 
introduces an effective damping term of approximately $\tau_{0} v_{F}^{2} \mathbf{q}^{2}$ for the collective modes. In order to stabilize the collective modes, the Hermitian part of Eq. (6) must be larger than the non-Hermitian part, or, equivalently, the eigenfrequency should be much larger than the damping rate. Since the gapped CPs are coupled to the Coulomb interaction, which dominates Eq. (6) in the long-wave limit, the conditions for the gapped CPs to be stable are (i) $1 / \tau_{v} \ll\left\{\left[e^{3}(\mathbf{q} \cdot \mathbf{B})\right] /\left(\epsilon_{0} \mathbf{q}^{2}\right)\right\}$ and (ii) $\tau_{0} v_{F}^{2} \mathbf{q}^{2} \ll$ $\left\{\left[e^{3}(\mathbf{q} \cdot \mathbf{B})\right] /\left(\epsilon_{0} \mathbf{q}^{2}\right)\right\}$. These two conditions are automatically satisfied in the long-wave limit, and hence, the gapped CPs are always stable against $\tau_{0}$. On the other hand, since the CZSs and gapless CPs are decoupled from the Coulomb interaction, as we show in the model below and prove generically in Appendix B, the conditions for CZSs and gapless CPs to be stable are (i) $\frac{1}{\tau_{v}} \ll\{e(\mathbf{q} \cdot \mathbf{B})[1+\beta(\mathbf{B}) f] /$ $[\beta(\mathbf{B})]\}$ and (ii) $\tau_{0} v_{F}^{2} \mathbf{q}^{2} \ll\{e(\mathbf{q} \cdot \mathbf{B})[1+\beta(\mathbf{B}) f] /[\beta(\mathbf{B})]\}$. These two conditions can be satisfied at some $\mathbf{q}$ only if

$$
\frac{\tau_{0}}{\tau_{v}} \ll \frac{e^{2} \mathbf{B}^{2}[1+\beta(\mathbf{B}) f]^{2}}{v_{F}^{2} \beta^{2}(\mathbf{B})} \sim \frac{\omega_{B}^{4}}{\mu^{4}}\left(1+\frac{\mu^{2} f}{2 \pi^{2} v_{F}^{3}}\right)^{2} .
$$

For simplicity, here we assume isotropic Fermi surfaces such that $\beta(\mathbf{B})=\left[\mu^{2} /\left(2 \pi^{2} v_{F}^{3}\right)\right]$. Thus, the upper bound of $\tau_{0}$ below which Eq. (6) is valid is given by Eq. (7).

Now let us analyze the (magnetic) point symmetry group of Eq. (6). Since the wave vector q enters Eq. (6) only through the $\mathbf{q} \cdot \mathbf{B}$ term, the symmetry group of Eq. (6) is much higher than the little group at $\mathbf{q}$. In fact, all the point group operations or combinations of point group operations and the time reversal that preserve $\mathbf{q} \cdot \mathbf{B}, \mathbf{B}$, and $f_{\nu, \nu^{\prime}}$ will keep Eq. (6) invariant. We emphasize that $\mathbf{q} \cdot \mathbf{B}$ is invariant under proper rotations and time reversal, but it changes sign under inversion, and $\mathbf{B}$ transforms as a vector under proper rotations, keeps invariant under the inversion, but changes sign under time reversal. Therefore, only two types of operations can leave Eq. (6) invariant: proper rotations with an axis parallel to $\mathbf{B}$ and time reversal followed by twofold proper rotations with an axis perpendicular to $\mathbf{B}$. In this paper, we denote the group consisting of these symmetry operations as $G_{c}(\mathbf{B})$, which is either a magnetic point group or a point group, depending on whether or not it contains combinations of a point group and the time-reversal operations. The solutions of Eq. (6) form the representations for the group $G_{c}(\mathbf{B})$, which can be divided into two categories: the trivial and nontrivial irreps. It is then easy to see that the $\mathrm{CP}$ solutions belong to the trivial irreps and the CZS solutions belong to the nontrivial ones. To be specific, as we prove in Appendix B, the multiplicity of the trivial irrep or the number of CPs is given by

$$
N_{\mathrm{CP}}(\mathbf{B})=\sum_{\nu}^{\prime} \frac{\left|G_{0}\right|\left|G_{c}(\mathbf{B}) \cap G_{\nu}\right|}{\left|G_{c}(\mathbf{B})\right|\left|G_{\nu}\right|},
$$

and the multiplicity of the nontrivial irreps or the number of CZSs is given by

$$
N_{\mathrm{CZS}}(\mathbf{B})=\sum_{\nu}^{\prime} \frac{\left|G_{0}\right|}{\left|G_{\nu}\right|}-\frac{\left|G_{0}\right|\left|G_{c}(\mathbf{B}) \cap G_{\nu}\right|}{\left|G_{c}(\mathbf{B})\right|\left|G_{\nu}\right|},
$$

where the summation of $\nu$ will be carried out over all inequivalent WPs. (Two WPs are equivalent if they are related by some symmetry operation.) $G_{0}$ is the maximal (magnetic) point group of the (magnetic) space group, $G_{\nu}$ is the subgroup of $G_{0}$ that leaves the $\nu$ th WP invariant, and $|G|$ is the number of elements in $G$. Here we take the Weyl semimetal TaAs [5] in space group $I 4{ }_{1} m d(\# 109)$ as an example to show the usage of Eqs. (8) and (9). Since TaAs is time-reversal symmetric and the maximal point group of $I 4_{1} m d$ is $C_{4 v}$, we obtain $G_{0}=C_{4 v}+T C_{4 v}$, where $T$ represents the time reversal. Totally, there are 24 different WPs in TaAs, which can be divided into two classes: eight WPs located at the $k_{z}=0$ plane and 16 WPs located off the $k_{z}=0$ plane. The WPs within the same class can be related by operations in $G_{0}$ and are considered to be equivalent from a symmetry point of view. The corresponding little groups that leave the WPs unchanged are $G_{1}=\{E\}$ and $G_{2}=\left\{E, T C_{2}\right\}$, respectively. Therefore, from Eq. (6), there are 24 independent variables in total leading to the same number of independent modes. Assuming the magnetic field is applied along the $C_{4}$ rotation axis, we obtain $G_{c}(\mathbf{B})=C_{4}$, and hence, $N_{\mathrm{CP}}=6$ and $N_{\mathrm{CZS}}=18$.

As we discuss above, in the semiclassical region we always have $\beta_{\nu}(\mathbf{B})-\beta_{\nu}(0) \sim\left(\omega_{B}^{2} / \mu^{2}\right) \beta_{\nu}(0)$, which is derived in detail in Appendix B. Thus, to the leadingorder effect of the magnetic field, we can omit the $\mathbf{B}$ dependence in $\beta_{\nu}(\mathbf{B})$. Then, Eq. (6) is in first order of $B$, and the corresponding symmetry group becomes higher than $G_{c}(\mathbf{B})$. This higher symmetry group denoted as $G_{c}(0)$ consists of all the proper rotations, time reversal (if present), and time reversal followed by proper rotations (if present) in the original group. Thus, $G_{c}(0)$ is nothing but the chiral subgroup of the little group at $\mathbf{q}=0$. Therefore, under semiclassical approximation, the number of CPs and CZSs [Eqs. (8) and (9)] should be calculated with $G_{c}(0)$ instead of $G_{c}(\mathbf{B})$.

\section{MINIMAL MODEL FOR CHIRAL ZERO SOUND}

At last, we consider a model Weyl semimetal system with only two pairs of WPs with point group symmetry $C_{2 v}$, as illustrated schematically in Fig. 1. For convenience, we split the valley index $\nu$ into a chirality index $s= \pm 1$ and a subvalley index $a=1,2$. Under the $C_{2}$ rotation, the $(s, 1)$ WP and the $(s, 2)$ WP transform to each other; under the $M_{x}$ mirror, the $(+, a) \mathrm{WP}$ and the $(-, a) \mathrm{WP}$ transform to each other. Thus, the representation matrices formed by $\eta_{\nu}$ can be written as $D_{s a ; s^{\prime} a^{\prime}}\left(C_{2}\right)=\tau_{a, a^{\prime}}^{x} \sigma_{s, s^{\prime}}^{0}$ and $D_{s a ; s^{\prime} a^{\prime}}\left(M_{x}\right)=$ $\tau_{a, a^{\prime}}^{0} \sigma_{s, s^{\prime}}^{x}$, where $\tau^{x, y, z}$ and $\sigma^{x, y, z}$ are Pauli matrices in the chirality space and subvalley space, respectively, and $\tau^{0}$ and $\sigma^{0}$ are two-by-two identity matrices. In the following, we 
omit the matrix subscripts for brevity. Without loss of generality, we choose the form of residual interaction as $f=f_{0} \tau^{0} \sigma^{0}+f_{1} \tau^{x} \sigma^{0}+f_{2} \tau^{0} \sigma^{x}+f_{3} \tau^{x} \sigma^{x}$, where we set $f_{0} \geq f_{1} \pm\left(f_{2}-f_{3}\right)$ and $f_{0} \geq-f_{1} \pm\left(f_{2}+f_{3}\right)$ to ensure that the interaction is positive semidefinite. The magnetic field is applied in the $z$ direction. Applying the representation matrices to Eq. (6), one can easily verify that the $C_{2}$ symmetry is kept but the $M_{x}$ symmetry is broken. Thus, the solutions will form the irreps of $C_{2}$. By diagonalizing Eq. (6), we obtain two branches of CPs

$$
\omega^{(1,2)}(\mathbf{q})+\frac{i}{\tau_{v}}= \pm \frac{e\left(q_{z} B\right)}{4 \pi^{2} \beta(B)} \sqrt{\zeta_{0}^{2}(\mathbf{q})-\zeta_{1}^{2}(\mathbf{q})},
$$

where $\zeta_{0}(\mathbf{q})=1+\beta(B)\left[f_{0}+f_{1}+2 e^{2} /\left(\epsilon_{0} \mathbf{q}^{2}\right)\right]$ and $\zeta_{1}(\mathbf{q})=$ $\beta(B)\left[f_{2}+f_{3}+2 e^{2} /\left(\epsilon_{0} \mathbf{q}^{2}\right)\right]$, and two branches of CZSs

$$
\omega^{(3,4)}(\mathbf{q})+\frac{i}{\tau_{v}}= \pm \frac{e\left(q_{z} B\right)}{4 \pi^{2} \beta(B)} \sqrt{\xi_{0}^{2}-\xi_{1}^{2}},
$$

where $\xi_{0}=1+\beta(B)\left(f_{0}-f_{1}\right)$ and $\xi_{1}=\beta(B)\left(f_{2}-f_{3}\right)$. In the long-wave limit, we have $\zeta_{0}^{2}(\mathbf{q})-\zeta_{1}^{2}(\mathbf{q}) \approx\left[\left(4 e^{2}\right) /\right.$ $\left.\left(\epsilon_{0} \mathbf{q}^{2}\right)\right]\left[1+\beta(B)\left(f_{0}+f_{1}-f_{2}-f_{3}\right)\right]$, so the CP modes are gapped and the plasmon frequency is approximately

$$
\frac{e^{2}\left(q_{z} B\right)}{2 \pi^{2}|\mathbf{q}|} \sqrt{\frac{1+\beta(B)\left(f_{0}+f_{1}-f_{2}-f_{3}\right)}{\beta(B) \epsilon_{0}}} .
$$

On the other hand, the CZS modes have linear dispersions along the magnetic field direction with the sound velocity

$$
c(B)=\frac{e B}{4 \pi^{2} \beta(B)} \sqrt{\xi_{0}^{2}-\xi_{1}^{2}} .
$$

Here we give a rough estimation of the sound velocity for a typical Weyl semimetal system. For simplicity, we set $f=0, B=10 \mathrm{~T}, \mu=30 \mathrm{meV}, v_{F}=2 \mathrm{eV} \AA \hbar^{-1}$; then we obtain $c(B) \approx 0.34 \mathrm{eV} \AA \hbar^{-1} \approx 5 \times 10^{4} \mathrm{~m} / \mathrm{s}$.

The eigenvectors of the two $\mathrm{CP}$ modes are

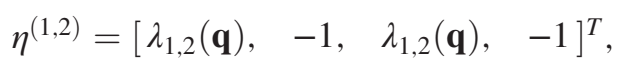

where $\lambda_{1,2}(\mathbf{q})=\left[\zeta_{0}(\mathbf{q}) \pm \sqrt{\zeta_{0}^{2}(\mathbf{q})-\zeta_{1}^{2}(\mathbf{q})}\right] / \zeta_{1}(\mathbf{q})$, and the eigenvectors of the two CZS modes are

$$
\eta^{(3,4)}=\left[\begin{array}{llll}
\lambda_{3,4}, & -1, & -\lambda_{3,4}, & 1
\end{array}\right]^{T},
$$

where $\lambda_{3,4}=\left(\xi_{0} \pm \sqrt{\xi_{0}^{2}-\xi_{1}^{2}}\right) / \xi_{1}$. In the above expressions, the bases of the $\eta$ vector are ordered as $(s, a)=(+, 1),(-, 1),(+, 2),(-, 2) \cdot \eta^{(1,2)}$ are invariant under $C_{2}$ and hence form the trivial irrep, whereas $\eta^{(3,4)}$ will change sign under $C_{2}$ and hence form the nontrivial irrep. The CP mode $\eta^{(1)}$ and the CZS mode $\eta^{(3)}$ are schematically plotted in Figs. 1(a) and 1(d), respectively. We can find clearly from Fig. 1 that the $\mathrm{CP}$ is such a mode that the quasiparticle densities with the same chirality oscillate with the same phase, while the quasiparticle densities with the opposite chiralities oscillate with opposite phases. Since the CME current from the $\nu$ th valley $\mathbf{j}_{\nu}$ is proportional to $\chi_{\nu} \eta_{\nu}$, a net current oscillation will be generated by the $\mathrm{CP}$ mode, which couples to the long-range Coulomb interaction and leads to a finite plasmon frequency in the long-wavelength limit. In contrast, in the CZS mode the valley densities with the same chirality oscillate with opposite phases, leading to the exact cancellation of CME currents from differen valleys. Therefore, the CZS mode will be completely decoupled from the charge dynamics and can keep its acoustic nature in the long-wavelength limit.

It is insightful to compare the possibility to have zero sound modes in ordinary metals and Weyl semimetals under magnetic field. The collective modes for the former metals have been discussed in detail in Ref. [43]. Using the description developed above for an ordinary metal, all the collective modes can be derived from the dynamics of Fermi-surface d.o.f. $\widetilde{\delta n_{\nu}}(\mathbf{r}, \mathbf{k}, t)$, which describe the small deviation of the quasiparticle occupation at the Fermi surface. For a system with approximately the sphere symmetry, it can be expanded using the sphere harmonics $Y_{\mathrm{LM}}\left(\theta_{k}, \phi_{k}\right)$. Therefore, the longitudinal mode is formed by the proper linear combination of the sphere harmonics with $m=0$ and becomes the plasmon mode. The transverse modes are described by the sphere harmonics with $m \neq 0$. Among them, the channel with $m= \pm 1$ will be absorbed into the Maxwell equation to describe the possible electrical magnetic wave, which contains no solution for frequency below the plasmon edge. Therefore, the only possible channels to have zero sound modes in an ordinary metal system are the channels with $|m| \geq 2$, provided that the effective residual interaction in these channels is positive definite to survive the Landau damping. These conditions are difficult to fulfill and so is zero sound in ordinary metal. Therefore, for Weyl semimetals under magnetic field, the CME provides a unique mechanism to stabilize the CZS with any form of residual interaction that does not cause instability. At least in the chiral limit, the dynamics of CZS involves only the anomalous current but not the normal current, and hence, it is free of Landau damping.

\section{THERMAL PROPERTIES OF CHIRAL ZERO SOUND}

The existence of the CP and CZS in the chiral limit leads to several interesting physical phenomena under the external magnetic field. Here we introduce two of them. The first one is the CZS contribution to the specific heat. The CZS modes can be viewed as a set of 1D collective modes dispersing only along the magnetic field. As we derive in Appendix $\mathrm{G}$, the specific heat contributed by the CZS is $\kappa(B, T) \approx k_{B}^{2} T \Lambda^{2} /[6 c(B)]$ for temperature $T \ll \Theta_{\mathrm{CZS}}$, where $\Theta_{\mathrm{CZS}}=c(B) \Lambda / k_{B}$ is the corresponding Debye temperature for the CZS, $c(B)$ is the sound velocity [Eq. (13)], 

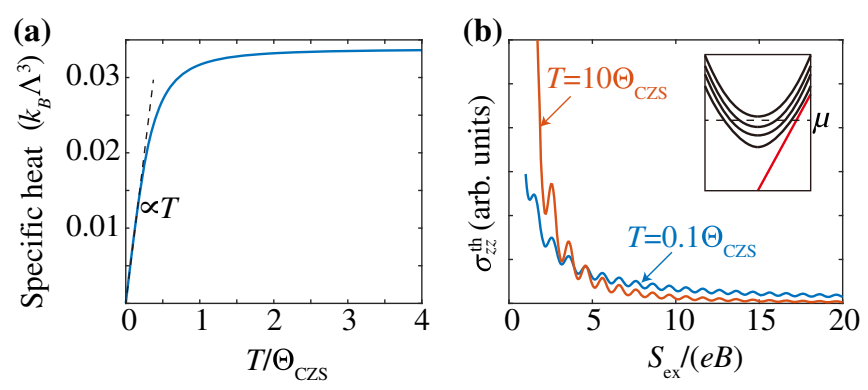

FIG. 2. (a) The specific heat (per unit volume) in the four-WPs model is plotted as a function of temperature. The specific heat is plotted in the unit of $k_{B} \Lambda^{3}$, where $k_{B}$ is Boltzmann's constant, and $\Lambda$ is the cutoff in the momentum integral. The temperature $T$ is plotted in units of Debye temperature for the CZS mode, $\Theta_{\mathrm{CZS}}=c(B) \Lambda / k_{B}$, where $c(B)$ is the speed of the CZS. (b) The thermal conductivity in the four-WPs model is plotted as a function of magnetic field. Here, $S_{\mathrm{ex}}(\mu)$ is the area enclosed by the extreme circle on the Fermi surface that is perpendicular to the magnetic field. The parameters are set as $\omega_{B}=0.2 \mu$, $k_{B} T=1 /\left(2 \tau_{0}\right)=0.001 \mu$, and $T / \Theta_{\mathrm{CZS}}=10$ and 0.1 for the blue line and red line, respectively.

$\Lambda$ is the momentum space cutoff, and $k_{B}$ is the Boltzmann constant, while in the high-temperature region $\left(T \gg \Theta_{\mathrm{CZS}}\right)$, the specific heat is $\kappa(B, T) \approx k_{B} \Lambda^{3} /\left(3 \pi^{2}\right)$. To be specific, in Fig. 2(a) we plot the specific heat as a function of temperature using some typical parameters for the Weyl semimetal systems. Although such a temperature dependence is similar to the quasiparticle contribution to the specific heat, the two can be distinguished from each other by their different field dependence. Another unusual property caused by the CZS is the thermal conductivity. Since the CZS disperses only along the field direction, the thermal current carried by the CZS modes can flow only along this direction. As a result, the thermal conductivity tensor contributed by the CZS modes has only one nonzero entry. As we derive in Appendix G, if the magnetic field is applied along the $z$ direction, the thermal conductivity is given by $\sigma_{i j}^{\text {th }}=\delta_{i, z} \delta_{j, z} \tau_{s}(T) c^{2}(B) \kappa(B, T)$, where $\tau_{s}(T)$ is the relaxation time for the CZS excitations. In the weak field and low-temperature region $\left(\tau_{0} \omega_{B} \ll 1, T \ll \Theta_{\mathrm{CZS}}\right)$, as $\kappa(B, T) \propto T / c(B)$ and $c(B) \propto B$, we obtain $\sigma_{z z}^{\text {th }} \propto T B$. In the weak field and high-temperature region $\left(\tau_{0} \omega_{B} \ll 1\right.$, $\left.T \gg \Theta_{\mathrm{CZS}}\right)$, as $\kappa \sim$ const, we obtain $\sigma_{z z}^{\text {th }} \propto B^{2}$.

In order to discuss the specific heat and thermal conductivity in the strong field region $\left(\tau_{0} \omega_{B} \gtrsim 1\right)$, we need to rederive the dynamic equation under the strong field, where the electronic states are already Landau levels. In this case, since the compressibility oscillates with the field, as a consequence, the velocity of the CZS as well as the thermal conductivity, in general, should also oscillate with the field. Here we focus only on the case $\omega_{B} \ll \mu$ so that there are still a large number of Landau levels below the chemical potential. As we introduce in Appendix H, it turns out that the dynamic equation has the same form of Eq. (6), except that the field dependence of the compressibility is modified. As we calculate in Appendix I, the compressibility in the strong field can be expressed as $\beta(B)=$ $\beta^{(0)}(B)+\beta^{(1)}(B)+\cdots$, where the $\beta^{(l \geq 1)}(B)$ terms oscillate as the $l$ th harmonics of $1 / B$. Under finite temperature, the ratio between the first and zeroth components is approximately

$$
\frac{\beta^{(1)}(B)}{\beta^{(0)}(B)} \approx \frac{\omega_{B}}{\mu} \frac{\exp \left(-\pi \frac{\mu}{\omega_{B}^{2} \tau_{0}}\right)}{\operatorname{sinch}\left(2 \pi^{2} \frac{\mu k_{B} T}{\omega_{B}^{2}}\right)} \cos \left(\frac{S_{\mathrm{ex}, \nu}(\mu)}{e B}-\frac{\pi}{4}\right),
$$

where $\operatorname{sinch}(x)=\left(e^{x}-e^{-x}\right) /(2 x)$, and $S_{\text {ex }}(\mu)$ is the area enclosed by the extreme circle (perpendicular to $\mathbf{B}$ ) on the Fermi surface. Here we assume $e B>0$ and $\mu>0$. Because of Eqs. (6) and (13), the oscillation in $\beta(B)$ will lead to the oscillation in the sound velocity of the CZS. Substituting Eq. (16) for Eq. (13), we obtain the first-order oscillation of the sound velocity as

$$
c(B) \approx c^{(0)}(B)\left(1-\frac{\xi_{0}}{\xi_{0}^{2}-\xi_{1}^{2}} \frac{\beta^{(1)}(B)}{\beta^{(0)}(B)}\right),
$$

where $c^{(0)}(B)$ is the nonoscillating component of the sound velocity. As both the specific heat and thermal conductivity are functions of the sound velocity, the oscillation in the velocity leads to oscillations in the specific heat and thermal conductivity as well. As an example, in Fig. 2(b) we plot the thermal conductivity as a function of magnetic field. In normal metals, the thermal conductivity is mainly contributed by electrons and acoustic phonons. The phonon part couples only indirectly to the magnetic field and usually does not change much with the field. Therefore, the part that oscillates with the field is mainly contributed by the free electrons in the normal metal, which satisfies the Wiedemann-Frantz law. As we introduce above, for the Weyl semimetals in the chiral limit, since the CZS can propagate only along the magnetic field, the thermal conductivity along the field will be contributed by both the CZS and free electrons leading to the dramatic violation of the Wiedemann-Frantz law, which is absent for thermal conductivity along the perpendicular direction. Early theoretical studies of the electronic contribution to the thermal conductivity in Weyl semimetal without considering the CZS modes obtain the $\mathbf{B}^{2}$ dependence for the thermal conductivity under magnetic field [53], which is quite different from the contribution from the CZS introduced above. Such a field-dependent violation of the WiedemannFrantz law has already been seen in the thermal conductivity measurement of TaAs under a magnetic field, indicating the possible contribution from the CZS [54]. We note that for realistic systems which are not deeply in the chiral limit, the CZS will also acquire nonzero velocity along the transverse direction of the magnetic field as well, which is caused by the accompanying normal current during the oscillation. Therefore, the CZS or the gapless $\mathrm{CP}$ can also contribute to the thermal conductivity along the 
transverse direction, but the effect should be much less by orders of magnitude than that of the longitudinal direction.

\section{DISCUSSION AND SUMMARY}

The above-mentioned quantum oscillations in specific heat and thermal conductivity can be viewed as strong evidence for the existence of the CZS but still indirect. It will be more convincing if we can also have direct ways to measure it. In this regard, the direct ultrasonic measurement of these materials under magnetic field and low temperature may be difficult but worth trying. Another possible experiment is inelastic neutron-scattering spectrum. Although the corresponding scattering cross section for electrons may be very small, the existence of the CZS can still be inferred from the spectrum of certain phonon modes, which have the same symmetry representation as the CZS and can hybridize with it when they intersect each other at some particular wave vector to form the "polariton mode".

In summary, we propose that an exotic collective mode, the chiral zero sound, can exist in a Weyl semimetal under magnetic field with the chiral limit, where the intervalley scattering time is much longer than the intravalley one. The CZS can propagate along the external magnetic field with its velocity being proportional to the field strength in the weak field limit and oscillating in the strong field. The CZS can lead to several interesting phenomena, among which the giant quantum oscillation in thermal conductivity is the most striking and can be viewed as "smoking gun" evidence for the existence of it.

\section{ACKNOWLEDGMENTS}

Z.S. and X.D. acknowledge financial support from the Hong Kong Research Grants Council (Project No. GRF16300918). Z. S. also acknowledges the Department of Energy Grant No. desc-0016239, the National Science Foundation EAGER Grant No. DMR 1643312, Simons Investigator Grants No. 404513, No. ONR N00014-14-1-0330, and No. NSF-MRSECDMR DMR 1420541, the Packard Foundation Grant No. 201665128, and the Schmidt Fund for Development of Majorana Fermions funded by the Eric and Wendy Schmidt Transformative Technology Fund.

\section{APPENDIX A: BOLTZMANN'S EQUATION AND COLLECTIVE MODES}

Let us first derive the Boltzmann equation, which applies when the Landau-level splitting, i.e., $\omega_{B}=v_{F} \sqrt{e B}$, is smaller than the imaginary part of the quasiparticle selfenergy and the chemical potential $\mu$. The semiclassical equations of motion of the Weyl fermion are $[49,50]$

$$
\begin{gathered}
\dot{\mathbf{k}}=-\partial_{\mathbf{r}} \epsilon(\mathbf{k}, \mathbf{r}, t)+e \dot{\mathbf{r}} \times \mathbf{B}, \\
\dot{\mathbf{r}}=\partial_{\mathbf{k}} \epsilon(\mathbf{k}, \mathbf{r}, t)-\dot{\mathbf{k}} \times \mathbf{\Omega}(\mathbf{k}),
\end{gathered}
$$

where $e=-|e|(|e|)$ is the electronlike (holelike) quasiparticle, and

$$
\boldsymbol{\Omega}(\mathbf{k})=-i\left\langle\partial_{\mathbf{k}} u(\mathbf{k})|\times| \partial_{\mathbf{k}} u(\mathbf{k})\right\rangle
$$

is the Berry curvature. The decoupled equations are

$$
\begin{aligned}
\gamma(\mathbf{k}, \mathbf{B}) \dot{\mathbf{k}}= & -\partial_{\mathbf{r}} \epsilon(\mathbf{k}, \mathbf{r}, t)+e \partial_{\mathbf{k}} \epsilon(\mathbf{k}, \mathbf{r}, t) \times \mathbf{B} \\
& +e\left[\partial_{\mathbf{r}} \epsilon(\mathbf{k}, \mathbf{r}, t) \cdot \mathbf{B}\right] \mathbf{\Omega}(\mathbf{k}), \\
\gamma(\mathbf{k}, \mathbf{B}) \dot{\mathbf{r}}= & \partial_{\mathbf{k}} \epsilon(\mathbf{k}, \mathbf{r}, t)+\partial_{\mathbf{r}} \epsilon(\mathbf{k}, \mathbf{r}, t) \times \mathbf{\Omega}(k) \\
& -e\left[\partial_{\mathbf{k}} \epsilon(\mathbf{k}, \mathbf{r}, t) \cdot \mathbf{\Omega}(\mathbf{k})\right] \mathbf{B},
\end{aligned}
$$

where

$$
\gamma(\mathbf{k}, \mathbf{B})=1-e \mathbf{B} \cdot \mathbf{\Omega}(\mathbf{k})
$$

is the phase-space measure. Now we denote the distribution function over phase space as $\rho(\mathbf{k}, \mathbf{r}, t)$, and due to particle number conservation, we have

$$
\begin{aligned}
& \rho(\mathbf{k}+d t \dot{\mathbf{k}}, \mathbf{r}+d t \dot{\mathbf{r}}, t+d t)\left(1+d t \partial_{\mathbf{k}} \cdot \dot{\mathbf{k}}+d t \partial_{\mathbf{r}} \cdot \dot{\mathbf{r}}\right) d^{3} \mathbf{k} d^{3} \mathbf{r} \\
& \quad=\rho(\mathbf{k}, \mathbf{r}, t) d^{3} \mathbf{k} d^{3} \mathbf{r},
\end{aligned}
$$

and hence,

$$
\begin{aligned}
0= & \frac{\partial}{\partial t} \rho(\mathbf{k}, \mathbf{r}, t)+\left[\left(\partial_{\mathbf{k}} \cdot \dot{\mathbf{k}}\right)+\dot{\mathbf{k}} \cdot \partial_{\mathbf{k}}+\partial_{\mathbf{r}} \cdot \dot{\mathbf{r}}+\dot{\mathbf{r}} \cdot \partial_{\mathbf{r}}\right] \\
& \times \rho(\mathbf{k}, \mathbf{r}, t) \\
= & \frac{\partial}{\partial t} \rho(\mathbf{k}, \mathbf{r}, t)+\partial_{\mathbf{k}} \cdot[\dot{\mathbf{k}} \rho(\mathbf{k}, \mathbf{r}, t)]+\partial_{\mathbf{r}} \cdot[\dot{\mathbf{r}} \rho(\mathbf{k}, \mathbf{r}, t)] .
\end{aligned}
$$

Here we neglect the scattering term in Boltzmann's equation.

From now on, we assume there are a few valleys and label the quantities in different valleys with a subscript $\nu$. For each valley, we introduce a weighted distribution function $n_{\nu}(\mathbf{k}, \mathbf{r}, t)=\rho_{\nu}(\mathbf{k}, \mathbf{r}, t) / \gamma(\mathbf{k})$, then the multivalley Boltzmann equation is given by

$$
\begin{aligned}
0= & \gamma_{\nu}(\mathbf{k}, \mathbf{B}) \frac{\partial}{\partial t} n_{\nu}(\mathbf{k}, \mathbf{r}, t)+\left\{-\partial_{\mathbf{r}} \epsilon_{\nu}(\mathbf{k}, \mathbf{r}, t)\right. \\
& \left.+e \partial_{\mathbf{k}} \epsilon_{\nu}(\mathbf{k}, \mathbf{r}, t) \times \mathbf{B}+e\left[\partial_{\mathbf{r}} \epsilon_{\nu}(\mathbf{k}, \mathbf{r}, t) \cdot \mathbf{B}\right] \boldsymbol{\Omega}_{\nu}(\mathbf{k})\right\} \\
& \cdot \partial_{\mathbf{k}} n_{\nu}(\mathbf{k}, \mathbf{r}, t)+\left\{\partial_{\mathbf{k}} \epsilon_{\nu}(\mathbf{k}, \mathbf{r}, t)+\partial_{\mathbf{r}} \epsilon_{\nu}(\mathbf{k}, \mathbf{r}, t)\right. \\
& \left.\times \boldsymbol{\Omega}_{\nu}(\mathbf{k})-e\left[\partial_{\mathbf{k}} \epsilon_{\nu}(\mathbf{k}, \mathbf{r}, t) \cdot \boldsymbol{\Omega}_{\nu}(\mathbf{k})\right] \mathbf{B}\right\} \cdot \partial_{\mathbf{r}} n_{\nu}(\mathbf{k}, \mathbf{r}, t),
\end{aligned}
$$

where, again, the scattering is neglected. In deriving Eq. (A9), we make use of the relations $\partial_{\mathbf{r}} \cdot\left[\gamma_{\nu}(\mathbf{k}, \mathbf{B}) \dot{\mathbf{r}}_{\nu}\right]=0$ and $\partial_{\mathbf{k}} \cdot\left[\gamma_{\nu}(\mathbf{k}, \mathbf{B}) \dot{\mathbf{k}}_{\nu}\right]=0$. Because of Eqs. (A4) and (A5), 
these two relations are satisfied as long as (i) $\mathbf{k}$ is not at the Weyl point, where the semiclassical method does not apply, and (ii) $\partial_{\mathbf{r}} \cdot \partial_{\mathbf{k}} \epsilon(\mathbf{k}, \mathbf{r}, t)=0$, which is automatically satisfied in our approximation for quasiparticle energy [Eq. (A13)].
In the presence of the collective mode, the single-particle energy $\epsilon_{\nu}(\mathbf{k}, \mathbf{r}, t)$ should be determined self-consistently. With quasiparticle excitation, the total energy is a functional of the distribution function [52]

$$
\begin{aligned}
E_{\text {total }}(t)= & E_{\text {total }}^{0}+\sum_{\nu} \int d^{3} \mathbf{r} \int \frac{d^{3} \mathbf{k}}{(2 \pi)^{3}} \gamma_{\nu}(\mathbf{k}, \mathbf{B}) \epsilon_{\nu}^{0}(\mathbf{k}) \delta n_{\nu}(\mathbf{k}, \mathbf{r}, t)+\frac{1}{2} \iint d^{3} \mathbf{r} d^{3} \mathbf{r}^{\prime} \frac{1}{\epsilon_{0}\left|\mathbf{r}-\mathbf{r}^{\prime}\right|} \delta n(\mathbf{r}, t) \delta n\left(\mathbf{r}^{\prime}, t\right) \\
& +\frac{1}{2} \sum_{\nu \nu^{\prime}} \int d^{3} \mathbf{r} \int \frac{d^{3} \mathbf{k}}{(2 \pi)^{3}} \gamma_{\nu}(\mathbf{k}, \mathbf{B}) \int \frac{d^{3} \mathbf{k}^{\prime}}{(2 \pi)^{3}} \gamma_{\nu^{\prime}}\left(\mathbf{k}^{\prime}, \mathbf{B}\right) f_{\nu, \nu^{\prime}} \delta n_{\nu}(\mathbf{k}, \mathbf{r}, t) \delta n_{\nu^{\prime}}\left(\mathbf{k}^{\prime}, \mathbf{r}, t\right),
\end{aligned}
$$

where the second and third terms denote, respectively, the long-range Coulomb and residual short-range interaction among the quasiparticles around the WPs. Here,

$$
\delta n_{\nu}(\mathbf{k}, \mathbf{r}, t)=n_{\nu}(\mathbf{k}, \mathbf{r}, t)-n_{F}\left[\epsilon_{\nu}^{0}(\mathbf{k})-\mu\right]
$$

is the deviation of distribution from equilibrium, $n_{F}(\epsilon)=$ $1 /\left\{1+\exp \left[-(\epsilon) /\left(k_{B} T\right)\right]\right\}$ is the Fermi-Dirac distribution,

$$
\delta n(\mathbf{r}, t)=\sum_{\nu} \int \frac{d^{3} \mathbf{k}}{(2 \pi)^{3}} \gamma_{\nu}(\mathbf{k}, \mathbf{B}) \delta n_{\nu}(\mathbf{k}, \mathbf{r}, t)
$$

is the charge density at position $\mathbf{r}$ and time $t$, and $f_{\nu, \nu^{\prime}}$ is a real matrix due to the Hermitian condition of the Hamiltonian. The $\mathbf{k}$ dependence of $f_{\nu, \nu^{\prime}}$ is neglected since we consider the case where the Fermi surfaces are very small compared to the Brillouin zone. The quasiparticle energy can then be derived as the functional derivation of the total energy

$$
\begin{aligned}
\epsilon_{\nu}(\mathbf{k}, \mathbf{r}, t)= & \epsilon_{\nu}^{0}(\mathbf{k})+\sum_{\nu^{\prime}} \int \frac{d^{3} \mathbf{k}^{\prime}}{(2 \pi)^{3}} f_{\nu, \nu^{\prime}} \gamma_{\nu^{\prime}}\left(\mathbf{k}^{\prime}, \mathbf{B}\right) \delta n_{\nu^{\prime}}\left(\mathbf{k}^{\prime}, \mathbf{r}, t\right) \\
& +e \varphi(\mathbf{r}, t),
\end{aligned}
$$

where $\varphi$ is the scalar potential determined by the Poisson equation

$$
-\partial_{\mathbf{r}}^{2} \varphi(\mathbf{r}, t)=\frac{e}{\epsilon_{0}} \delta n(\mathbf{r}, t) .
$$

Now we assume the deviation from equilibrium takes the form of the plane wave

$$
\delta n_{\nu}(\mathbf{k}, \mathbf{r}, t)=\delta n_{\nu}(\mathbf{k}) e^{i(\mathbf{q} \cdot \mathbf{r}-\omega t)} .
$$

Following this definition, we can rewrite the quasiparticle energy as

$$
\begin{aligned}
\epsilon_{\nu}(\mathbf{k}, \mathbf{r}, t)= & \epsilon_{\nu}^{0}(\mathbf{k})+\sum_{\nu^{\prime}} \int \frac{d^{3} \mathbf{k}^{\prime}}{(2 \pi)^{3}}\left(f_{\nu, \nu^{\prime}}+\frac{e^{2}}{\epsilon_{0} \mathbf{q}^{2}}\right) \\
& \times \gamma_{\nu^{\prime}}\left(\mathbf{k}^{\prime}, \mathbf{B}\right) \delta n_{\nu^{\prime}}\left(\mathbf{k}^{\prime}\right) e^{i(\mathbf{q} \cdot \mathbf{r}-\omega t)} .
\end{aligned}
$$

The equation of motion to first order of $\delta n_{\nu}(\mathbf{k})$ is given by

$$
\begin{aligned}
0= & -\gamma_{\nu}(\mathbf{k}, \mathbf{B}) \omega \delta n_{\nu}(\mathbf{k}) \\
& +\left\{\mathbf{q} \cdot \mathbf{v}_{\nu}(\mathbf{k})-e \mathbf{q} \cdot \mathbf{B}\left[\mathbf{v}_{\nu}(\mathbf{k}) \cdot \mathbf{\Omega}_{\nu}(\mathbf{k})\right]\right\}\left[\delta n_{\nu}(\mathbf{k})+\delta_{T}\left[\mu-\epsilon_{\nu}^{0}(\mathbf{k})\right] \sum_{\nu^{\prime}} \int \frac{d^{3} \mathbf{k}^{\prime}}{(2 \pi)^{3}}\left(f_{\nu, \nu^{\prime}}+\frac{e^{2}}{\epsilon_{0} \mathbf{q}^{2}}\right) \gamma_{\nu^{\prime}}\left(\mathbf{k}^{\prime}, \mathbf{B}\right) \delta n_{\nu^{\prime}}\left(\mathbf{k}^{\prime}\right)\right] \\
& -i e\left[\mathbf{v}_{\nu}(\mathbf{k}) \times \mathbf{B}\right] \cdot \partial_{\mathbf{k}} \delta n_{\nu}(\mathbf{k}),
\end{aligned}
$$

where $\mathbf{v}_{\nu}(\mathbf{k})=\partial_{\mathbf{k}} \epsilon_{\nu}^{0}(\mathbf{k})$ and $\delta_{T}(\epsilon)=-\partial_{\epsilon} n_{F}(\epsilon)$.

For convenience, we replace the $3 \mathrm{D}$ variable $\mathbf{k}$ in Eq. (A17) with an energy $\epsilon$ and a $2 \mathrm{D}$ wave vector $\boldsymbol{\sigma}$ on the energy surface. The integration over $\mathbf{k}$ in the $\nu$ th valley can be rewritten as

$$
\int d^{3} \mathbf{k}=\int_{0}^{\infty} d \epsilon \int_{\epsilon} d^{2} \boldsymbol{\sigma} \frac{1}{\left|\mathbf{v}_{\nu}(\epsilon, \boldsymbol{\sigma})\right|},
$$

where $\int_{\epsilon}$ means $\boldsymbol{\sigma}$ takes a value on the $2 \mathrm{D}$ surface with fixed energy $\epsilon$. Apparently, the solution of Eq. (A17) takes the form 


$$
\delta n_{\nu}(\mathbf{k})=-\partial_{\epsilon} n_{F}(\epsilon-\mu) \delta n_{\nu}(\boldsymbol{\sigma}),
$$

where $\boldsymbol{\sigma}$ takes a value on the Fermi surface. Integrating the energy, Eq. (A17) becomes

$$
\begin{aligned}
0= & -\frac{\gamma_{\nu}(\boldsymbol{\sigma}, \mathbf{B})}{\left|\mathbf{v}_{\nu}(\boldsymbol{\sigma})\right|} \omega \delta n_{\nu}(\boldsymbol{\sigma}) \\
& +\left\{\mathbf{q} \cdot \hat{\mathbf{v}}_{\nu}(\boldsymbol{\sigma})-e \mathbf{q} \cdot \mathbf{B}\left[\hat{\mathbf{v}}_{\nu}(\boldsymbol{\sigma}) \cdot \boldsymbol{\Omega}_{\nu}(\boldsymbol{\sigma})\right]\right\} \times\left[\delta n_{\nu}(\boldsymbol{\sigma})+\sum_{\nu^{\prime}} \int \frac{d^{2} \boldsymbol{\sigma}^{\prime}}{(2 \pi)^{3}}\left(f_{\nu, \nu^{\prime}}+\frac{e^{2}}{\epsilon_{0} \mathbf{q}^{2}}\right) \frac{\gamma_{\nu^{\prime}}\left(\boldsymbol{\sigma}^{\prime}, \mathbf{B}\right)}{\left|\mathbf{v}_{\nu^{\prime}}\left(\boldsymbol{\sigma}^{\prime}\right)\right|} \delta n_{\nu^{\prime}}\left(\boldsymbol{\sigma}^{\prime}\right)\right] \\
& -i e\left[\hat{\mathbf{v}}_{\nu}(\boldsymbol{\sigma}) \times \mathbf{B}\right] \cdot \partial_{\mathbf{k}} \delta n_{\nu}(\boldsymbol{\sigma}),
\end{aligned}
$$

where $\hat{v}_{\nu}(\boldsymbol{\sigma})=\mathbf{v}_{\nu}(\boldsymbol{\sigma}) /\left|\mathbf{v}_{\nu}(\boldsymbol{\sigma})\right|$ and

$$
\partial_{\mathbf{k}}=\mathbf{v}_{\nu}(\mathbf{k}) \frac{\partial}{\partial \epsilon}+\sum_{i=1,2} \partial_{\mathbf{k}} \boldsymbol{\sigma}_{i} \frac{\partial}{\partial \boldsymbol{\sigma}_{i}} .
$$

In Eq. (A20), all the quantities are defined on the Fermi surfaces, so we omit the energy dependence of these quantities; e.g., $\mathbf{v}_{\nu}(\boldsymbol{\sigma})$ is shorthand for $\mathbf{v}_{\nu}(\mu, \boldsymbol{\sigma})$.

\section{APPENDIX B: THE CHIRAL LIMIT}

We can decompose $\delta n$ into two parts: the first part $\overline{\delta n}$ changes particle numbers in different valleys, and the second part $\widetilde{\delta n}$ preserves the particle number in each valley but deform the shape of Fermi surface in each valley. We refer to $\overline{\delta n}$ as the valley d.o.f. and $\widetilde{\delta n}$ as the Fermi-surface d.o.f. In the general case, these two d.o.f. are strongly coupled. However, as we argue below, in the chiral limit, the dynamics of these two d.o.f. is decoupled. In the presence of a scattering term, $\delta n$ in general damps with time, but the valley degrees and the Fermi-surface degrees can have different relaxation times. We denote the relaxation time of $\widetilde{\delta n}$ as $\tau_{0}$, whereas the relaxation time of $\overline{\delta n}$ is $\tau_{v}$. Then the time derivative term in Eq. (A20) should be replaced by

$\omega \delta n_{\nu}(\boldsymbol{\sigma}) \rightarrow\left(\omega+\frac{i}{\tau_{v}}\right) \overline{\delta n}_{\nu}(\boldsymbol{\sigma})+\left(\omega+\frac{i}{\tau_{0}}\right) \widetilde{\delta n}_{\nu}(\boldsymbol{\sigma})$.

The chiral limit refers to the case where $\tau_{0}$ is much smaller $\tau_{v}$, i.e.,

$$
\frac{\tau_{0}}{\tau_{v}} \ll 1 .
$$

This limit can be achieved when the intravalley scattering is much stronger than the intervalley scattering. In Appendix E, we discuss the relaxation times contributed by impurity scattering. In the simple case in Appendix E, $\overline{\delta n}_{\nu}$ is defined as

$$
\overline{\delta n}_{\nu}=\frac{1}{\beta_{\nu}(\mathbf{B})} \int \frac{d^{2} \boldsymbol{\sigma}}{(2 \pi)^{3}} \frac{\gamma_{\nu}(\boldsymbol{\sigma}, \mathbf{B})}{\left|\mathbf{v}_{\nu}(\boldsymbol{\sigma})\right|} \delta n_{\nu}(\boldsymbol{\sigma}),
$$

where

$$
\beta_{\nu}(\mathbf{B})=\int \frac{d^{2} \boldsymbol{\sigma}}{(2 \pi)^{3}} \frac{\gamma_{\nu}(\boldsymbol{\sigma}, \mathbf{B})}{\left|\mathbf{v}_{\nu}(\boldsymbol{\sigma})\right|}=\frac{d \rho_{\nu}}{d \mu}
$$

is the compressibility of the $\nu$ th valley, and $\rho_{\nu}$ is total particle density of the $\nu$ th valley. Then, Eq. (A20) can be rewritten as

$$
\begin{aligned}
\frac{\gamma_{\nu}(\boldsymbol{\sigma}, \mathbf{B})}{\left|\mathbf{v}_{\nu}(\boldsymbol{\sigma})\right|}\left[\left(\omega+\frac{i}{\tau_{v}}\right) \overline{\delta n}_{\nu}+\left(\omega+\frac{i}{\tau_{0}}\right) \widetilde{\delta n}_{\nu}(\boldsymbol{\sigma})\right] \\
=+\left\{\mathbf{q} \cdot \hat{\mathbf{v}}_{\nu}(\boldsymbol{\sigma})-e \mathbf{q} \cdot \mathbf{B}\left[\hat{\mathbf{v}}_{\nu}(\boldsymbol{\sigma}) \cdot \boldsymbol{\Omega}_{\nu}(\boldsymbol{\sigma})\right]\right\} \\
\times\left[\delta n_{\nu}(\boldsymbol{\sigma})+\sum_{\nu^{\prime}}\left(f_{\nu, \nu^{\prime}}+\frac{e^{2}}{\epsilon_{0} \mathbf{q}^{2}}\right) \beta_{\nu^{\prime}}(\mathbf{B}) \overline{\delta n}_{\nu^{\prime}}\right] \\
-i e\left[\hat{\mathbf{v}}_{\nu}(\boldsymbol{\sigma}) \times \mathbf{B}\right] \cdot \partial_{\mathbf{k}} \delta n_{\nu}(\boldsymbol{\sigma}) .
\end{aligned}
$$

In the following, we study the physics in zeroth order of $\tau_{0}$ and leave the discussion on the finite $\tau_{0}$ effect for Appendix E. To zeroth order of $\tau_{0}$, the Fermi-surface d.o.f. are always in thermal equilibrium, i.e., $\widetilde{\delta n_{\nu}}(\boldsymbol{\sigma})=0$, and any deviation from equilibrium will be immediately killed by the strong scattering. By integrating $\boldsymbol{\sigma}$ into Eq. (B5), we get a generalized eigenvalue equation

$$
\begin{aligned}
\left(\omega+\frac{i}{\tau_{v}}\right) \chi_{\nu} \eta_{\nu}= & \frac{e(\mathbf{q} \cdot \mathbf{B})}{4 \pi^{2} \beta_{\nu}(\mathbf{B})} \eta_{\nu} \\
& +\frac{e}{4 \pi^{2}}(\mathbf{q} \cdot \mathbf{B}) \sum_{\nu^{\prime}}\left(f_{\nu, \nu^{\prime}}+\frac{e^{2}}{\epsilon_{0} \mathbf{q}^{2}}\right) \eta_{\nu^{\prime}} .
\end{aligned}
$$

Here, $\chi_{\nu}= \pm 1$ is the chirality of the $\nu$ th valley, and $\eta_{\nu}=$ $\beta_{\nu}(\mathbf{B}) \overline{\delta n}_{\nu}$ is the disequilibrium quasiparticle number in the $\nu$ th valley. In deriving Eq. (B6), we apply

$$
\int d^{2} \boldsymbol{\sigma} \hat{\mathbf{v}}_{\nu}(\boldsymbol{\sigma}) \cdot \boldsymbol{\Omega}_{\nu}(\boldsymbol{\sigma})=\int d \mathbf{S} \cdot \boldsymbol{\Omega}_{\nu}(\boldsymbol{\sigma})=-2 \pi \chi_{\nu}
$$

Now let us discuss the symmetry of Eq. (B6). Apparently, Eq. (B6) has a higher symmetry than the little group of $\mathbf{q}$ : It contains all the symmetries that preserve the chiralities of WPs and the direction of magnetic field. The direction of $\mathbf{q}$ is irrelevant to the symmetry. This is because 
in the chiral limit, the electric field proportional to $\mathbf{q}$ enters the equation only through the $\mathbf{q} \cdot \mathbf{B}$ term and thus couples only to the chiral d.o.f. Therefore, finite $\mathbf{q}$ breaks only the symmetries changing the chiralities. In the following, we denote the symmetry group of Eq. (B6) as $G_{c}(\mathbf{B})$. We emphasize that some antiunitary symmetry, like time reversal followed by a crystalline symmetry, can also keep the chiralities and the magnetic field invariant. And, since $f_{\nu, \nu^{\prime}}$ is a real matrix, these antiunitary symmetries act on Eq. (B6) as unitary operators. The explicit representation matrix of all these symmetries is given in Eq. (B9).

It should be noticed that, to leading order of magnetic field, i.e., setting $\beta_{\nu}(\mathbf{B})=\beta_{\nu}(0)$, Eq. (B6) even has a symmetry higher than $G_{c}(\mathbf{B})$ : The magnetic field enters the equation only through term $\mathbf{q} \cdot \mathbf{B}$; thus, the direction of magnetic field becomes irrelevant to the symmetry. We denote this higher symmetry group as $G_{c}(0)$, which consists of all the symmetries of the zero field system that do not change chirality.

The solutions of Eq. (B6) must form irreps of $G_{c}(\mathbf{B})$. As we show in the next two sections, the trivial irreps of $G_{c}(\mathbf{B})$ always couple to the charge-density oscillation, and thus, we call these modes forming trivial irreps CPs. As we prove, only two of the CPs are gapped, whereas other CPs are gapless in the long-wave limit. On the other hand, all the nontrivial irreps are decoupled from density oscillation, so we call them the CZSs. Now let us calculate the number of trivial irreps in the solution to Eq. (B6). We first consider a set of symmetry-related WPs in the inner Brillouin zone, and one of them has the little group $G_{W}$. We denote the maximal (magnetic) point group of the space group as $G_{0}$, then each symmetry-related WP can be represented by a coset representative of $G_{0} / G_{W}$,

$$
G_{0}=h_{1} G_{W}+h_{2} G_{W}+\cdots
$$

The representation formed by the valley degrees is given by

$$
\begin{gathered}
\forall g \in G_{0}, \quad D_{h, h^{\prime}}(g)= \begin{cases}1 & \text { if } g h^{\prime} \in h G_{W}, \\
0 & \text { else },\end{cases} \\
\operatorname{Tr} D(g)= \begin{cases}\left|G_{0}\right| /\left|G_{W}\right|, & g \in G_{W}, \\
0, & g \notin G_{W} .\end{cases}
\end{gathered}
$$

Now we reduce $D$ to irreps of $G_{c}(\mathbf{B})$. The number of trivial irreps is given by

$$
\frac{1}{\left|G_{c}(\mathbf{B})\right|} \sum_{g \in G_{c}(\mathbf{B})} \operatorname{Tr} D(g)=\frac{\left|G_{0}\right|\left|G_{c}(\mathbf{B}) \cap G_{W}\right|}{\left|G_{c}(\mathbf{B})\right|\left|G_{W}\right|} .
$$

Therefore, for a system with a few sets of nonequivalent WPs, the number of $\mathrm{CP}$ modes and CZS modes are given by

$$
N_{\mathrm{CP}}(\mathbf{B})=\sum_{\nu}^{\prime} \frac{\left|G_{0}\right|\left|G_{c}(\mathbf{B}) \cap G_{\nu}\right|}{\left|G_{c}(\mathbf{B})\right|\left|G_{\nu}\right|}
$$

and

$$
N_{\mathrm{CZS}}(\mathbf{B})=\sum_{\nu}^{\prime} \frac{\left|G_{0}\right|}{\left|G_{\nu}\right|}-\frac{\left|G_{0}\right|\left|G_{c}(\mathbf{B}) \cap G_{\nu}\right|}{\left|G_{c}(\mathbf{B})\right|\left|G_{\nu}\right|},
$$

respectively. Here, $\nu$ sums over all inequivalent WPs, and $G_{\nu}$ is the little group of the $\nu$ th WP.

\section{APPENDIX C: CZS}

If $\eta_{\nu}$ is not a trivial irrep of $G_{c}(\mathbf{B})$, then there must be $\sum_{\nu} \eta_{\nu}=0$, implying that it does not cause any chargedensity oscillation. Thus, for nontrivial irreps, we can omit the Coulomb term, and the corresponding modes are the CZSs. Now let us solve the equation of motion for the CZS. Notice that the matrix on the rhs of Eq. (B6) is real and symmetric, so we diagonalize it as

$$
f_{\nu, \nu^{\prime}}+\frac{1}{\beta_{\nu}(\mathbf{B})} \delta_{\nu, \nu^{\prime}}=\frac{1}{\bar{\beta}(\mathbf{B})} \sum_{a} O_{\nu, a} \lambda_{a} O_{\nu^{\prime}, a}
$$

where $\bar{\beta}(\mathbf{B})$ is the averaged $\beta_{\nu}(\mathbf{B}), O$ is an orthogonal matrix, and the $\lambda_{a}$ 's are dimensionless numbers. Applying the transformation

$$
\eta_{\nu}=\sum_{a} O_{\nu, a} \eta_{a}^{\prime}
$$

we can rewrite Eq. (B6) as

$$
\left(\omega+\frac{i}{\tau_{v}}\right) \sum_{\nu, a^{\prime}} O_{\nu, a} \chi_{\nu} O_{\nu, a^{\prime}} \eta_{a^{\prime}}^{\prime}=\frac{e}{4 \pi^{2}}(\mathbf{q} \cdot \mathbf{B}) \lambda_{a} \eta_{a}^{\prime} .
$$

Applying the transformation

$$
\Xi_{a, a^{\prime}}=\sum_{\nu} \frac{1}{\sqrt{\lambda_{a}}} O_{\nu, a} \chi_{\nu} O_{\nu, a^{\prime}} \frac{1}{\sqrt{\lambda_{a^{\prime}}}}, \quad \eta_{a}^{\prime \prime}=\sqrt{\lambda_{a}} \eta_{a}^{\prime},
$$

we get a regular eigenvalue problem

$$
\Xi \eta^{\prime \prime}=\frac{e(\mathbf{q} \cdot \mathbf{B})}{4 \pi^{2} \bar{\beta}(\mathbf{B})\left(\omega+\frac{i}{\tau_{v}}\right)} \eta^{\prime \prime}
$$

where $\Xi$ has the symmetry of $G_{c}(\mathbf{B})$. The dispersion of the CZS is given by

$$
\omega_{\mathrm{CZS}, n}(\mathbf{q})+\frac{i}{\tau_{v}}=\frac{e(\mathbf{q} \cdot \mathbf{B})}{4 \pi^{2} \bar{\beta}(\mathbf{B}) \xi_{n}}, \quad n=1, \ldots, N_{\mathrm{CZS}}(\mathbf{B}) .
$$

Here, $\xi_{n}$ is the $n$th eigenvalue of $\Xi$.

It should be noticed that $\Xi$ is real and symmetric (such that the $\xi_{n}$ 's are real) only if all $\lambda_{a}$ 's are positive. Thus, the number of CZS modes is given by Eq. (B13) only if Eq. (C1) is positive definite. Otherwise, only irreps where 
all $\lambda_{a}$ 's are positive correspond to physically observable modes. The irreps having negative $\lambda_{a}$ 's in general have complex $\xi$ and so are not stable.

\section{APPENDIX D: CP}

For the trivial irreps, in general we have $\sum_{\nu} \eta_{\nu} \neq 0$. Therefore, the trivial irreps contribute to density oscillation, and thus, the Coulomb term must be considered. However, as $\left[e^{2} /\left(\epsilon_{0} \mathbf{q}^{2}\right)\right]$ is a rank-one operator, in the long-wave limit, there should be only one channel that responds to Coulomb interaction. To separate this channel, we define the projection operator $P_{\nu, \nu^{\prime}}=\left(1 / N_{W}\right)$, where $N_{W}$ is the number of WPs, and divide the terms on the rhs of Eq. (B6) into four components:

$$
\begin{aligned}
{\left[\frac{e^{2}}{\epsilon_{0} \mathbf{q}^{2}}\right]+f+\left[\frac{1}{\beta(\mathbf{B})}\right]=} & P\left(\left[\frac{e^{2}}{\epsilon_{0} \mathbf{q}^{2}}\right]+f+\left[\frac{1}{\beta(\mathbf{B})}\right]\right) P+Q\left(f+\left[\frac{1}{\beta(\mathbf{B})}\right]\right) Q \\
& +P\left(f+\left[\frac{1}{\beta(\mathbf{B})}\right]\right) Q+Q\left(f+\left[\frac{1}{\beta(\mathbf{B})}\right]\right) P .
\end{aligned}
$$

Here, $\left[e^{2} /\left(\epsilon_{0} \mathbf{q}^{2}\right)\right]$ represents the matrix where every element is $\left[e^{2} /\left(\epsilon_{0} \mathbf{q}^{2}\right)\right],\{1 /[\beta(\mathbf{B})\}$ represents the diagonal matrix $\left\{1 /\left[\beta_{\nu}(\mathbf{B})\right]\right\} \delta_{\nu, \nu^{\prime}}$, and $Q=\mathbb{I}-P$, where $\mathbb{I}$ is the identity matrix. We apply an orthogonal transformation $V=\mathbb{I}+S-S^{T}$ where $S=P S Q$, to remove the mixing term between the $P$ and $Q$ subspaces. To second order of $\mathbf{q}$, we find that

$$
S=-\frac{\epsilon_{0}}{e^{2} N_{W}} \mathbf{q}^{2} P\left(f+\left[\frac{1}{\beta(\mathbf{B})}\right]\right) Q+\mathcal{O}\left(\mathbf{q}^{4}\right)
$$

and

$$
V^{T}\left(\left[\frac{e^{2}}{\epsilon_{0} \mathbf{q}^{2}}\right]+f+\left[\frac{1}{\beta(\mathbf{B})}\right]\right) V=P\left(\left[\frac{e^{2}}{\epsilon_{0} \mathbf{q}^{2}}\right]+f+\left[\frac{1}{\beta(\mathbf{B})}\right]\right) P+Q\left(f+\left[\frac{1}{\beta(\mathbf{B})}\right]\right) Q+\mathcal{O}\left(\mathbf{q}^{2}\right) .
$$

Using the fact $\sum_{\nu^{\prime \prime}} V_{\nu^{\prime \prime}, \nu} \chi_{\nu^{\prime \prime}} V_{\nu^{\prime \prime}, \nu^{\prime}}=\chi_{\nu} \delta_{\nu, \nu^{\prime}}+\mathcal{O}\left(\mathbf{q}^{2}\right)$, we can rewrite Eq. (B6) as

$$
\left(\omega+\frac{1}{\tau_{v}}\right) \chi_{\nu} \eta_{\nu}=\sum_{\nu^{\prime}}\left\{P\left(\left[\frac{e^{2}}{\epsilon_{0} \mathbf{q}^{2}}\right]+f+\left[\frac{1}{\beta(\mathbf{B})}\right]\right) P+Q\left(f+\left[\frac{1}{\beta(\mathbf{B})}\right]\right) Q\right\}_{\nu, \nu^{\prime}} \eta_{\nu^{\prime}}+\mathcal{O}\left(\mathbf{q}^{2}\right) .
$$

To solve this generalized eigenvalue equation, we apply the technique used in Appendix C: diagonalizing the matrix on the rhs and transforming the equation to a regular eigenvalue problem. Let us write the matrix on the righthand side as $\{1 /[\bar{\beta}(\mathbf{B})]\} \sum_{a} O_{\nu, a} \lambda_{a} O_{\nu^{\prime}, a}$. Applying the transformation

$\Xi_{a, a^{\prime}}=\sum_{\nu} \frac{1}{\sqrt{\lambda_{a}}} O_{\nu, a} \chi_{\nu} O_{\nu, a^{\prime}} \frac{1}{\sqrt{\lambda_{a^{\prime}}}}, \quad \eta_{a}^{\prime \prime}=\sqrt{\lambda_{a}} \sum_{\nu} O_{\nu, a} \eta_{\nu}$,

we get a regular eigenvalue problem

$$
\Xi \eta^{\prime \prime}=\frac{e(\mathbf{q} \cdot \mathbf{B})}{4 \pi^{2} \bar{\beta}(\mathbf{B})\left(\omega+\frac{i}{\tau_{v}}\right)} \eta^{\prime \prime}
$$

The frequencies of the CP modes are then given by

$$
\omega_{\mathrm{CP}, n}+\frac{i}{\tau_{v}}=\frac{e(\mathbf{q} \cdot \mathbf{B})}{4 \pi^{2} \bar{\beta}(\mathbf{B}) \xi_{n}(\mathbf{q})}
$$

where the $\xi_{n}$ 's are eigenvalues of $\Xi$. Now let us analyze the spectrum of $\Xi$. For convenience, we set $O_{\nu, 1}$ as the eigenvector of $P$, so the corresponding eigenvalue is

$$
\begin{aligned}
\lambda_{1}= & \frac{e^{2} N_{W}}{\epsilon_{0} \mathbf{q}^{2}} \bar{\beta}(\mathbf{B})+\frac{1}{N_{W}} \sum_{\nu, \nu^{\prime}}\left(f_{\nu, \nu^{\prime}} \bar{\beta}(\mathbf{B})+\frac{\bar{\beta}(\mathbf{B})}{\beta_{\nu}(\mathbf{B})} \delta_{\nu, \nu^{\prime}}\right) \\
& +\mathcal{O}\left(\mathbf{q}^{2}\right),
\end{aligned}
$$

which is singular in the limit $\mathbf{q} \rightarrow 0$. Then, due to Eq. (D12), the $\Xi$ matrix takes the form

$$
\Xi=\left(\begin{array}{cc}
0 & \zeta \\
\zeta^{T} & \Xi^{\prime}
\end{array}\right)+\mathcal{O}\left(\mathbf{q}^{2}\right)
$$

where

$$
\zeta_{1, a}=\Xi_{1, a}, \quad a=2,3 \ldots
$$

and

$$
\Xi_{a, a^{\prime}}^{\prime}=\Xi_{a, a^{\prime}}, \quad a, a^{\prime}=2,3 \ldots
$$


are submatrices of $\Xi$. We emphasize that for $a \geq 2, \lambda_{a}$ is not singular. Thus, in the limit $\mathbf{q} \rightarrow 0, \Xi^{\prime}$ approaches a constant matrix, whereas $\zeta_{1, a} \sim|\mathbf{q}|$. Therefore, by diagonalizing $\Xi^{\prime}$, we can rewrite $\Xi$ as

$\Xi=U^{T}\left(\begin{array}{cccc}0 & c_{2}|\mathbf{q}| & c_{3}|\mathbf{q}| & \ldots \\ c_{2}|\mathbf{q}| & \xi_{2}^{\prime} & 0 & \ldots \\ c_{3}|\mathbf{q}| & 0 & \xi_{3}^{\prime} & \ldots \\ \vdots & \vdots & \vdots & \ddots\end{array}\right) U+\mathcal{O}\left(\mathbf{q}^{2}\right)$

where the $\xi_{a}^{\prime}$ 's are eigenvalues of $\Xi^{\prime}$, and $U$ is some orthogonal matrix. Now we prove that one of the $\xi_{a=2,3 \ldots}^{\prime}$ is zero. We denote the diagonal matrix $\delta_{\nu, \nu^{\prime}} \chi_{\nu}$ as $[\chi]$. Then, the projected $[\chi]$ matrix in $Q$ subspace is $Q[\chi] Q=[\chi]-$ $P[\chi]-[\chi] P$. Apparently, $\eta_{\nu}=1$ and $\eta_{\nu}=\chi_{\nu}$ are two zero eigenvectors of $Q[\chi] Q$, wherein $\eta_{\nu}=1$ is in subspace $P$, whereas $\eta_{\nu}=\chi_{\nu}$ is in subspace $Q$. As $\Xi^{\prime}$ is equivalent to $Q[\chi] Q$ up to an invertible transformation, $\Xi^{\prime}$ has one zero eigenvalue in the $Q$ subspace. Therefore, one of the $\xi_{a=2,3 \ldots}^{\prime}$ is zero. Here we choose $\xi_{2}^{\prime}=0$. In the limit $\mathbf{q} \rightarrow 0$, we have the $\Xi$ eigenvalues as

$$
\begin{array}{r}
\xi_{1}(\mathbf{q})=-\xi_{2}(\mathbf{q})=\left|c_{2} \| \mathbf{q}\right|+\mathcal{O}\left(\mathbf{q}^{2}\right), \\
\xi_{n}(\mathbf{q})=\xi_{n}^{\prime}(\mathbf{q})+\mathcal{O}\left(\mathbf{q}^{2}\right), \quad n=3, \ldots, N_{\mathrm{CP}}(\mathbf{B}) .
\end{array}
$$

Therefore, due to Eq. (D18), $n=1,2$ correspond to the gapped CP modes, whereas $n=3, \ldots, N_{\mathrm{CP}}(\mathbf{B})$ correspond to the gapless CP modes. The low-energy behavior of the gapless CPs is very similar with the CZSs: Both of them have a linear dispersion in the limit $\mathbf{q} \rightarrow 0$. However, a vital difference is that gapless CPs are coupled to gapped CPs through the $c_{a \geq 3}$ terms in Eq. (D12), whereas the CZSs are not. As a result, the dispersions of gapless and gapped CPs form anticrossings, whereas the dispersions of CZSs and gapped CPs form symmetry-protected crossings.

Here we give a simplified method to calculate the gapped $\mathrm{CP}$ frequency. Since the gapped CP is driven by the Coulomb interaction, for simplicity, in this method we omit $f_{\nu, \nu^{\prime}}$ and $1 / \tau_{v}$. From Eq. (B6), we get

$$
\eta_{\nu}=\frac{\frac{e}{4 \pi^{2}}(\mathbf{q} \cdot \mathbf{B})}{\chi_{\nu} \beta_{\nu}(\mathbf{B}) \omega-\frac{e}{4 \pi^{2}}(\mathbf{q} \cdot \mathbf{B})} \cdot \frac{e^{2}}{\epsilon_{0} \mathbf{q}^{2}} \sum_{\nu^{\prime}} \eta_{\nu^{\prime}}
$$

and thus,

$$
1=\frac{e^{2}}{\epsilon_{0} \mathbf{q}^{2}} \sum_{\nu} \frac{\frac{e}{4 \pi^{2}}(\mathbf{q} \cdot \mathbf{B}) \beta_{\nu}(\mathbf{B})}{\chi_{\nu} \beta_{\nu}(\mathbf{B}) \omega-\frac{e}{4 \pi^{2}}(\mathbf{q} \cdot \mathbf{B})} .
$$

Supposing $\omega$ is a constant in the limit $\mathbf{q} \rightarrow 0$, we have

$$
1=\frac{e^{2}}{\epsilon_{0} \mathbf{q}^{2}} \sum_{\nu}\left(\chi_{\nu} \frac{\frac{e}{4 \pi^{2}}(\mathbf{q} \cdot \mathbf{B})}{\omega}+\frac{\left(\frac{e}{4 \pi^{2}} \mathbf{q} \cdot \mathbf{B}\right)^{2}}{\beta_{\nu}(\mathbf{B}) \omega^{2}}+\chi_{\nu} \frac{\left(\frac{e}{4 \pi^{2}} \mathbf{q} \cdot \mathbf{B}\right)^{3}}{\beta_{\nu}^{2}(\mathbf{B}) \omega^{3}}+\frac{\left(\frac{e}{4 \pi^{2}} \mathbf{q} \cdot \mathbf{B}\right)^{4}}{\beta_{\nu}^{3}(\mathbf{B}) \omega^{4}}+\cdots\right)
$$

To zeroth order of $\mathbf{q}$, we need keep only the first two terms in the above equation. The first term must vanish due to the no-go theorem of Weyl semimetals [55,56], which says $\sum_{\nu} \chi_{\nu}=0$. Thus, we have

$$
\omega_{\mathrm{CP}, 1,2}(\mathbf{q} \rightarrow 0)= \pm \frac{e^{2}}{4 \pi^{2}}|\hat{\mathbf{q}} \cdot \mathbf{B}| \sqrt{\frac{1}{\epsilon_{0}} \sum_{\nu} \frac{1}{\beta_{\nu}(\mathbf{B})}},
$$

where $\hat{\mathbf{q}}=\mathbf{q} /|\mathbf{q}|$.

\section{APPENDIX E: FINITE INTRAVALLEY SCATTERING}

In this Appendix, we solve Eq. (B5) to first order of $\tau_{0}$ and justify the chiral limit approximation using secondorder perturbation theory. First, let us derive $\tau_{0}$ and $\tau_{v}$ explicitly in terms of the scattering cross section. We model the scattering cross section as

$$
W_{\nu, \nu^{\prime}}\left(\boldsymbol{\sigma}, \boldsymbol{\sigma}^{\prime}\right)=\delta_{\nu, \nu^{\prime}} w_{0}+\left(1-\delta_{\nu, \nu^{\prime}}\right) w_{1}
$$

Thus, the scattering term is given by

$$
\begin{aligned}
\mathcal{S}\left[n_{\nu}(\boldsymbol{\sigma})\right]= & \sum_{\nu^{\prime}} \int \frac{d^{2} \boldsymbol{\sigma}^{\prime}}{(2 \pi)^{3}} \frac{\gamma_{\nu^{\prime}}\left(\boldsymbol{\sigma}^{\prime}, \mathbf{B}\right)}{\left|\mathbf{v}_{\nu^{\prime}}\left(\boldsymbol{\sigma}^{\prime}\right)\right|} W_{\nu, \nu^{\prime}}\left(\boldsymbol{\sigma}, \boldsymbol{\sigma}^{\prime}\right) \\
& \times\left[\delta n_{\nu^{\prime}}\left(\boldsymbol{\sigma}^{\prime}\right)-\delta n_{\nu}(\boldsymbol{\sigma})\right] \\
= & \left(w_{0}-w_{1}\right) \int \frac{d^{2} \boldsymbol{\sigma}^{\prime}}{(2 \pi)^{3}} \frac{\gamma_{\nu}\left(\boldsymbol{\sigma}^{\prime}, \mathbf{B}\right)}{\left|\mathbf{v}_{\nu}\left(\boldsymbol{\sigma}^{\prime}\right)\right|}\left[\delta n_{\nu}\left(\boldsymbol{\sigma}^{\prime}\right)-\delta n_{\nu}(\boldsymbol{\sigma})\right] \\
& +w_{1} \sum_{\nu^{\prime}} \int \frac{d^{2} \boldsymbol{\sigma}^{\prime}}{(2 \pi)^{3}} \frac{\gamma_{\nu^{\prime}}\left(\boldsymbol{\sigma}^{\prime}, \mathbf{B}\right)}{\left|\mathbf{v}_{\nu^{\prime}}\left(\boldsymbol{\sigma}^{\prime}\right)\right|}\left[\delta n_{\nu^{\prime}}\left(\boldsymbol{\sigma}^{\prime}\right)-\delta n_{\nu}(\boldsymbol{\sigma})\right] .
\end{aligned}
$$

We introduce the quasiparticle d.o.f.

$$
\overline{\delta n}_{\nu}=\frac{1}{\beta_{\nu}(\mathbf{B})} \int \frac{d^{2} \boldsymbol{\sigma}}{(2 \pi)^{3}} \frac{\gamma_{\nu}(\boldsymbol{\sigma}, \mathbf{B})}{\left|\mathbf{v}_{\nu}(\boldsymbol{\sigma}, \mathbf{B})\right|} \delta n_{\nu}(\boldsymbol{\sigma})
$$

and $\widetilde{\delta n_{\nu}}(\boldsymbol{\sigma})=\delta n_{\nu}(\boldsymbol{\sigma})-\overline{\delta n}_{\nu}$. Then, Eq. (E2) can be rewritten as 


$$
\begin{aligned}
\mathcal{S}\left[n_{\nu}(\boldsymbol{\sigma})\right] & =\left(w_{0}-w_{1}\right)\left[\beta_{\nu}(\mathbf{B}) \overline{\delta n}_{\nu}-\beta_{\nu}(\mathbf{B}) \delta n_{\nu}(\boldsymbol{\sigma})\right]+w_{1} \sum_{\nu^{\prime}}\left[\beta_{\nu^{\prime}}(\mathbf{B}) \overline{\delta n}_{\nu^{\prime}}-\beta_{\nu^{\prime}}(\mathbf{B}) \delta n_{\nu}(\boldsymbol{\sigma})\right] \\
& =-\left(w_{0}-w_{1}\right) \beta_{\nu}(\mathbf{B}) \widetilde{\delta n_{\nu}}(\boldsymbol{\sigma})+w_{1} \sum_{\nu^{\prime}} \beta_{\nu^{\prime}}(\mathbf{B})\left(\overline{\delta n}_{\nu^{\prime}}-\overline{\delta n}_{\nu}\right)-w_{1} \sum_{\nu^{\prime}} \beta_{\nu^{\prime}}(\boldsymbol{B}) \widetilde{\delta n_{\nu}}(\mathbf{B}) \\
& =-\left(w_{0} \beta_{\nu}(\mathbf{B})+w_{1} \sum_{\nu^{\prime} \neq \nu} \beta_{\nu^{\prime}}(\mathbf{B})\right) \overline{\delta n}_{\nu}(\boldsymbol{\sigma})-\left(w_{1} \sum_{\nu^{\prime}} \beta_{\nu^{\prime}}(\mathbf{B})\right) \overline{\delta n}_{\nu}(\boldsymbol{\sigma})+w_{1} \sum_{\nu^{\prime}} \beta_{\nu^{\prime}}(\mathbf{B}) \overline{\delta n}_{\nu^{\prime}} .
\end{aligned}
$$

Defining

$$
\begin{gathered}
\frac{1}{\tau_{0, \nu}}=w_{0} \beta_{\nu}(\mathbf{B})+w_{1} \sum_{\nu^{\prime} \neq \nu} \beta_{\nu^{\prime}}(\mathbf{B}), \\
\frac{1}{\tau_{v}}=w_{1} \sum_{\nu^{\prime}} \beta_{\nu^{\prime}},
\end{gathered}
$$

we can rewrite the scattering term as

$$
\mathcal{S}\left[\delta n_{\nu}(\boldsymbol{\sigma})\right]=-\frac{\widetilde{\delta n_{\nu}}(\boldsymbol{\sigma})}{\tau_{0, \nu}}-\frac{\overline{\delta n}_{\nu}}{\tau_{v}}+w_{1} \sum_{\nu^{\prime}} \beta_{\nu^{\prime}}(\mathbf{B}) \overline{\delta n}_{\nu^{\prime}} .
$$

The first term relaxes the deformation of the Fermi surfaces that do not change the quasiparticle number in each valley, the second term relaxes the quasiparticle number in each valley, and the last term is feedback from the change of the total quasiparticle number. Because the scattering term is elastic, the total quasiparticle number on the Fermi surface should be a constant under the scattering. One can confirm this by observing $\sum_{\nu} \int\left[\left(d^{2} \boldsymbol{\sigma}\right) /(2 \pi)^{3}\right]\left[\gamma_{\nu}(\boldsymbol{\sigma}, \mathbf{B}) /\right.$ $\left.\left|\mathbf{v}_{\nu}(\boldsymbol{\sigma}, \mathbf{B})\right|\right] \mathcal{S}\left[n_{\nu}(\boldsymbol{\sigma})\right]=0$. For simplicity, in the following we neglect the $\nu$ dependence in $\tau_{0, \nu}$; i.e., we set $\tau_{0, \nu}=\tau_{0}$.

For simplicity, here we consider isotropic Fermi surfaces, where $\left|\mathbf{v}_{\nu}(\boldsymbol{\sigma})\right|, \beta_{\nu}(\boldsymbol{\sigma}), \boldsymbol{\Omega}_{\nu}(\boldsymbol{\sigma}) \cdot \hat{v}_{\nu}(\boldsymbol{\sigma})$ do not depend on $\boldsymbol{\sigma}$, and $\gamma_{\nu}(\boldsymbol{\sigma}, \mathbf{B})=1$. According to Eq. (B5), to leading order of $\tau_{0}$, we get the $\widetilde{\delta n_{\nu}}(\boldsymbol{\sigma})$ part as

$$
\widetilde{\delta n_{\nu}}(\boldsymbol{\sigma})=-i \tau_{0}\left[\mathbf{q} \cdot \mathbf{v}_{\nu}(\boldsymbol{\sigma})\right] \frac{\left(\omega+\frac{i}{\tau_{v}}\right) \chi_{\nu}}{\frac{e}{4 \pi^{2}}(\mathbf{q} \cdot \mathbf{B})} \eta_{\nu}+\mathcal{O}\left(\tau_{0}^{2}\right) .
$$

Now we look at the leading-order effect of $\tau_{0}$ on the CZS modes. For a specific branch of the CZS, Eq. (E8) gives

$$
\widetilde{\delta n}_{\nu}(\boldsymbol{\sigma})=-i \tau_{0}\left[\mathbf{q} \cdot \mathbf{v}_{\nu}(\boldsymbol{\sigma})\right] \frac{\chi_{\nu}}{\bar{\beta}(\mathbf{B}) \xi} \eta_{\nu}+\mathcal{O}\left(\tau_{0}^{2}\right),
$$

where $\xi$ is the corresponding eigenvalue of the $\Xi$ matrix [Eq. (C4)]. Substituting it back into Eq. (B5) and integrating $\boldsymbol{\sigma}$, we get

$$
\begin{aligned}
\left(\omega+\frac{i}{\tau_{v}}\right) \chi_{\nu} \eta_{\nu}= & -i \tau_{0} \frac{\left\{\left(\mathbf{q} \cdot \mathbf{v}_{F, \nu}\right)^{2}\right\}}{\xi} \eta_{\nu}+\frac{e(\mathbf{q} \cdot \mathbf{B})}{4 \pi^{2} \beta_{\nu}(\mathbf{B})} \eta_{\nu} \\
& +\frac{e}{4 \pi^{2}}(\mathbf{q} \cdot \mathbf{B}) \sum_{\nu^{\prime}}\left(f_{\nu, \nu^{\prime}}+\frac{e^{2}}{\epsilon_{0} \mathbf{q}^{2}}\right) \eta_{\nu^{\prime}},
\end{aligned}
$$

where

$$
\left\{\left(\mathbf{q} \cdot \mathbf{v}_{F, \nu}\right)^{2}\right\}=\frac{1}{\bar{\beta}(\mathbf{B})} \int \frac{d^{2} \boldsymbol{\sigma}}{(2 \pi)^{3}} \frac{1}{\left|\mathbf{v}_{\nu}(\boldsymbol{\sigma})\right|}\left[\mathbf{q} \cdot \mathbf{v}_{\nu}(\boldsymbol{\sigma})\right]^{2} .
$$

Apparently, finite $\tau_{0}$ introduces a non-Hermitian term in the dynamic equation of $\eta$. This term leads to a damping rate proportional to $\tau_{0} q^{2} v_{F}^{2} / \xi$. Therefore, for zero sound to be stable, the following relation should be satisfied:

$$
\frac{e B q}{\beta \xi} \gg \tau_{0} \frac{q^{2} v_{F}^{2}}{\xi} .
$$

Considering the intervalley scattering, the following relation should also be satisfied:

$$
\frac{e B q}{\beta \xi} \gg \frac{1}{\tau_{v}} .
$$

The above two inequalities are equivalent to

$$
\frac{\xi}{\tau_{v} v_{F}} \frac{\mu^{2}}{\omega_{B}^{2}} \ll q \ll \frac{1}{\tau_{0} v_{F}} \frac{\omega_{B}^{2}}{\mu^{2}},
$$

which have solutions only if

$$
\frac{\tau_{0}}{\tau_{v}} \ll \frac{\omega_{B}^{4}}{\mu^{4}} \frac{1}{\xi} .
$$

Equation (E15) gives the upper limit of $\tau_{0}$, above which the CZS modes become unstable. It should be noticed that $1 / \xi$ is of the order of $1+\bar{\beta}(\mathbf{B})|f|$.

Perturbation theory for gapless $\mathrm{CP}$ modes is similar to the perturbation theory for the CZS modes, and the stable condition of the gapless $\mathrm{CP}$ modes is also given by Eq. (E15). Now we consider the gapped CP modes. For the positive branch of the gapped $\mathrm{CP}$, the frequency of which is denoted as $\omega_{\mathrm{CP}}$, Eq. (E8) gives

$$
\widetilde{\delta n_{\nu}}=-i \tau_{0}\left[\hat{\mathbf{q}} \cdot \mathbf{v}_{\nu}(\boldsymbol{\sigma})\right] \frac{\omega_{\mathrm{CP}} \chi_{\nu}}{\frac{e}{4 \pi^{2}}(\hat{\mathbf{q}} \cdot \mathbf{B})} \eta_{\nu}+\mathcal{O}\left(\tau_{0}^{2}\right) .
$$

Following the above analysis, we find this term leads to a damping rate proportional to $\bar{\beta}(\mathbf{B})\left(q v_{F}\right)\left(\tau_{0} \omega_{\mathrm{CP}}\right) /$ $e B \sim\left(q v_{F}\right)\left(\tau_{0} \omega_{\mathrm{CP}}\right)\left(\mu^{2} / \omega_{B}^{2}\right)$. Thus, for the gapped CP to be stable, there should be 


$$
\tau_{0} \ll \frac{1}{q v_{F}} \frac{\omega_{B}^{2}}{\mu^{2}} .
$$

Therefore, the $\mathrm{CP}$ modes in the long-wave limit are always stable against the intravalley scattering.

\section{APPENDIX F: $k$-DEPENDENT SCATTERING AND INTERACTION}

In this section, we consider the $\mathbf{k}$ dependence in the scattering cross section and residual short-range interaction. We show that the dynamic equations (6) and (B6) are still correct in the chiral limit, except that the parameters should be modified.

\section{Elastic scattering conserving the renormalized energy}

We emphasize that it is the renormalized quasiparticle energy, other than the bare quasiparticle energy, that is conserved in the scattering process. This effect is not considered in Appendixes A-E. As we explain below, when the short-range interaction $f_{\nu, \nu^{\prime}}\left(\mathbf{k}, \mathbf{k}^{\prime}\right)$ is $\mathbf{k}$ independent, this effect can be neglected safely. However, when $f_{\nu, \nu^{\prime}}\left(\mathbf{k}, \mathbf{k}^{\prime}\right)$ becomes $\mathbf{k}$ dependent, it is crucial to consider this effect to obtain the correct dynamic equation.

In the presence of a $\mathbf{k}$-dependent interaction, the renormalized quasiparticle energy in Eq. (A16) is modified to

$$
\begin{aligned}
\epsilon_{\nu}(\mathbf{k})= & \epsilon_{\nu}^{0}(\mathbf{k})+\sum_{\nu^{\prime}} \int \frac{d^{3} \mathbf{k}^{\prime}}{(2 \pi)^{3}}\left(f_{\nu, \nu^{\prime}}\left(\mathbf{k}, \mathbf{k}^{\prime}\right)+\frac{e^{2}}{\epsilon_{0} \mathbf{q}^{2}}\right) \\
& \times \gamma_{\nu^{\prime}}\left(\mathbf{k}^{\prime}, \mathbf{B}\right) \delta n_{\nu^{\prime}}\left(\mathbf{k}^{\prime}\right) .
\end{aligned}
$$

Now we neglect the $\mathbf{r}$ and $t$ dependence in $\delta n_{\nu}(\mathbf{k})$ because the scattering process has a much shorter length scale and timescale than the collective mode. Here we omit the planewave factor $e^{i(\mathbf{q} \cdot \mathbf{r}-\omega t)}$ for simplicity. Changing $\mathbf{k}$ to the variable $\epsilon, \boldsymbol{\sigma}$ and writing $n_{\nu}(\mathbf{k})$ as $n_{\nu}(\epsilon, \boldsymbol{\sigma})=n_{F}(\epsilon-\mu)+$ $\delta(\epsilon-\mu) \delta n_{\nu}(\boldsymbol{\sigma})$ (as we introduce in Appendix A), we can write the correction to the quasiparticle energies of the quasiparticles on the Fermi surface as

$$
\epsilon_{\nu}^{0}(\mathbf{k})=\mu \Rightarrow \Delta_{\nu}(\boldsymbol{\sigma})=\epsilon_{\nu}(\mathbf{k})-\epsilon_{\nu}^{0}(\mathbf{k})=\sum_{\nu^{\prime}} \int \frac{d^{2} \boldsymbol{\sigma}^{\prime}}{(2 \pi)^{3}}\left(f_{\nu, \nu^{\prime}}\left(\boldsymbol{\sigma}, \boldsymbol{\sigma}^{\prime}\right)+\frac{e^{2}}{\epsilon_{0} \mathbf{q}^{2}}\right) \frac{\gamma_{\nu^{\prime}}\left(\mathbf{k}^{\prime}, \mathbf{B}\right)}{\left|\mathbf{v}_{\nu^{\prime}}\left(\boldsymbol{\sigma}^{\prime}\right)\right|} \delta n_{\nu}\left(\boldsymbol{\sigma}^{\prime}\right) .
$$

We require the renormalized quasiparticle energy to be conserved in the scattering process. Thus, the scattering term is modified to

$$
\mathcal{S}\left[n_{\nu}(\mathbf{k})\right]=\sum_{\nu^{\prime}} \iint \frac{d \epsilon^{\prime} d^{2} \boldsymbol{\sigma}^{\prime}}{(2 \pi)^{3}} \frac{\gamma_{\nu}\left(\boldsymbol{\sigma}^{\prime}, \mathbf{B}\right)}{\left|\mathbf{v}_{\nu}\left(\boldsymbol{\sigma}^{\prime}\right)\right|} W_{\nu, \nu^{\prime}}\left(\boldsymbol{\sigma}, \boldsymbol{\sigma}^{\prime}\right) \delta\left[\epsilon+\Delta_{\nu}(\boldsymbol{\sigma})-\epsilon^{\prime}-\Delta_{\nu^{\prime}}\left(\boldsymbol{\sigma}^{\prime}\right)\right]\left[n_{\nu^{\prime}}\left(\epsilon^{\prime}, \boldsymbol{\sigma}^{\prime}\right)-n_{\nu}(\epsilon, \boldsymbol{\sigma})\right] .
$$

To linear order of $\delta n_{\nu}(\boldsymbol{\sigma})$, we obtain

$$
\begin{aligned}
\mathcal{S}\left[n_{\nu}(\mathbf{k})\right]= & \sum_{\nu^{\prime}} \iint \frac{d \epsilon^{\prime} d^{2} \boldsymbol{\sigma}^{\prime}}{(2 \pi)^{3}} \frac{\gamma_{\nu}\left(\boldsymbol{\sigma}^{\prime}, \mathbf{B}\right)}{\left|\mathbf{v}_{\nu}\left(\boldsymbol{\sigma}^{\prime}\right)\right|} W_{\nu, \nu^{\prime}}\left(\boldsymbol{\sigma}, \boldsymbol{\sigma}^{\prime}\right) \delta\left(\epsilon-\epsilon^{\prime}\right)\left[\delta\left(\epsilon^{\prime}-\mu\right) \delta n_{\nu^{\prime}}\left(\boldsymbol{\sigma}^{\prime}\right)-\delta(\epsilon-\mu) \delta n_{\nu}(\boldsymbol{\sigma})\right] \\
& +\sum_{\nu^{\prime}} \iint \frac{d \epsilon^{\prime} d^{2} \boldsymbol{\sigma}^{\prime} \gamma_{\nu}\left(\boldsymbol{\sigma}^{\prime}, \mathbf{B}\right)}{(2 \pi)^{3}} \frac{\mathbf{v}_{\nu}\left(\boldsymbol{\sigma}^{\prime}\right) \mid}{\mid} W_{\nu, \nu^{\prime}}\left(\boldsymbol{\sigma}, \boldsymbol{\sigma}^{\prime}\right)\left\{n_{F}\left[\epsilon+\Delta_{\nu}(\boldsymbol{\sigma})-\Delta_{\nu^{\prime}}\left(\boldsymbol{\sigma}^{\prime}\right)-\mu\right]-n_{F}(\epsilon-\mu)\right\} \\
= & \delta(\epsilon-\mu) \sum_{\nu^{\prime}} \int \frac{d^{2} \boldsymbol{\sigma}^{\prime}}{(2 \pi)^{3}} \frac{\gamma_{\nu}\left(\boldsymbol{\sigma}^{\prime}, \mathbf{B}\right)}{\left|\mathbf{v}_{\nu}\left(\boldsymbol{\sigma}^{\prime}\right)\right|} W_{\nu, \nu^{\prime}}\left(\boldsymbol{\sigma}, \boldsymbol{\sigma}^{\prime}\right)\left[\delta n_{\nu^{\prime}}\left(\boldsymbol{\sigma}^{\prime}\right)+\Delta_{\nu^{\prime}}\left(\boldsymbol{\sigma}^{\prime}\right)-\delta n_{\nu}(\boldsymbol{\sigma})-\Delta_{\nu}(\boldsymbol{\sigma})\right] .
\end{aligned}
$$

\section{The valley degrees of freedom}

In Appendix B, we decompose $\delta n_{\nu}(\boldsymbol{\sigma})$ into two parts: the valley d.o.f. $\overline{\delta n}_{\nu}(\boldsymbol{\sigma})$ and the Fermi-surface d.o.f. $\widetilde{\delta n_{\nu}}(\boldsymbol{\sigma})$. In Appendix E, we show that if the short-range interaction and the scattering cross section are $\mathbf{k}$ independent, $\overline{\delta n}_{\nu}(\boldsymbol{\sigma})$ is a constant for each valley [Eq. (E3)]. In the following, we show that with $k$-dependent interaction and scattering, the valley d.o.f. are still well defined but their form is modified.
First, we decompose the scattering cross section into an intravalley component and an intervalley component

$$
W_{\nu, \nu^{\prime}}\left(\boldsymbol{\sigma}, \boldsymbol{\sigma}^{\prime}\right)=\delta_{\nu, \nu^{\prime}} W_{\nu}^{(0)}\left(\boldsymbol{\sigma}, \boldsymbol{\sigma}^{\prime}\right)+\left(1-\delta_{\nu, \nu^{\prime}}\right) W_{\nu \nu^{\prime}}^{(1)}\left(\boldsymbol{\sigma}, \boldsymbol{\sigma}^{\prime}\right) .
$$

Correspondingly, we then decompose the scattering term into an intravalley term $\mathcal{S}^{(0)}$ and an intervalley term $\mathcal{S}^{(1)}$. Here, we are interested only in $\mathcal{S}^{(0)}$, 


$$
\mathcal{S}^{(0)}\left[\delta n_{\nu}(\boldsymbol{\sigma})\right]=\frac{1}{\lambda} \int \frac{d^{2} \boldsymbol{\sigma}^{\prime}}{(2 \pi)^{3}} \frac{\gamma_{\nu}\left(\boldsymbol{\sigma}^{\prime}, \mathbf{B}\right)}{\left|\mathbf{v}_{\nu}\left(\boldsymbol{\sigma}^{\prime}\right)\right|} W_{\nu}^{(0)}\left(\boldsymbol{\sigma}, \boldsymbol{\sigma}^{\prime}\right)\left[\delta n_{\nu^{\prime}}\left(\boldsymbol{\sigma}^{\prime}\right)+\Delta_{\nu^{\prime}}\left(\boldsymbol{\sigma}^{\prime}\right)-\delta n_{\nu}(\boldsymbol{\sigma})-\Delta_{\nu}(\boldsymbol{\sigma})\right]
$$

The valley d.o.f. are undamped under the intravalley scattering. The following condition is sufficient and necessary for $\overline{\delta n}_{\nu}(\boldsymbol{\sigma})$ to be undamped under arbitrary intravalley scattering

$$
\overline{\delta n}_{\nu}(\boldsymbol{\sigma})+\Delta_{\nu}(\boldsymbol{\sigma})=\overline{\delta n}_{\nu}(\boldsymbol{\sigma})+\sum_{\nu^{\prime}} \int \frac{d^{2} \boldsymbol{\sigma}^{\prime}}{(2 \pi)^{3}}\left(f_{\nu, \nu^{\prime}}\left(\boldsymbol{\sigma}, \boldsymbol{\sigma}^{\prime}\right)+\frac{e^{2}}{\epsilon_{0} \mathbf{q}^{2}}\right) \frac{\gamma_{\nu^{\prime}}\left(\mathbf{k}^{\prime}, \mathbf{B}\right)}{\left|\mathbf{v}_{\nu^{\prime}}\left(\boldsymbol{\sigma}^{\prime}\right)\right|} \overline{\delta n}_{\nu^{\prime}}\left(\boldsymbol{\sigma}^{\prime}\right)=a_{\nu}
$$

where $a_{\nu}$ is some constant. It is direct to see that when $f$ is independent of $\boldsymbol{\sigma}, \mathrm{Eq}$. (E3) satisfies Eq. (F7).

We expand the valley d.o.f. on a set of basis functions

$$
\overline{\delta n}_{\nu}(\boldsymbol{\sigma})=\sum_{\alpha} c_{\alpha} h_{\nu \alpha}(\boldsymbol{\sigma})
$$

For $\mathbf{k}$-independent $f$, we can simply set $h_{\nu \alpha}(\boldsymbol{\sigma})=\delta_{\nu \alpha}$, such that for arbitrary $c_{\alpha}$, Eq. (F7) is satisfied. For $k$-dependent $f$, we require $h_{\nu \alpha}$ to satisfy

$$
\begin{aligned}
& h_{\nu \alpha}(\boldsymbol{\sigma})+\sum_{\nu^{\prime}} \int \frac{d^{2} \boldsymbol{\sigma}^{\prime}}{(2 \pi)^{3}}\left(f_{\nu, \nu^{\prime}}\left(\boldsymbol{\sigma}, \boldsymbol{\sigma}^{\prime}\right)+\frac{e^{2}}{\epsilon_{0} \mathbf{q}^{2}}\right) \frac{\gamma_{\nu^{\prime}}\left(\mathbf{k}^{\prime}, \mathbf{B}\right)}{\left|\mathbf{v}_{\nu^{\prime}}\left(\boldsymbol{\sigma}^{\prime}\right)\right|} \\
& \times h_{\nu^{\prime} \alpha}\left(\boldsymbol{\sigma}^{\prime}\right)=A_{\nu \alpha},
\end{aligned}
$$

where $A_{\nu \alpha}$ is a matrix, such that for arbitrary $c_{\alpha}$, Eq. (F7) is satisfied, and $a_{\nu}$ is given as

$$
a_{\nu}=\sum_{\alpha} c_{\alpha} A_{\nu \alpha}
$$

We decompose the short-range interaction into a k-independent part $\bar{f}$ and a k-dependent part $\delta f$,

$$
f_{\nu, \nu^{\prime}}\left(\boldsymbol{\sigma}, \boldsymbol{\sigma}^{\prime}\right)=\bar{f}_{\nu, \nu^{\prime}}+\delta f_{\nu, \nu^{\prime}}\left(\boldsymbol{\sigma}, \boldsymbol{\sigma}^{\prime}\right)
$$

Then, the basis functions subject to Eq. (F9) can be solved by series expansion in order of $\delta f$. We take the trial solution

$$
h_{\nu \alpha}(\boldsymbol{\sigma})=\delta_{\nu \alpha}+\sum_{m=1}^{\infty} h_{\nu \alpha}^{(m)}
$$

where $h^{(m)}$ is in $m$ th order of $\delta f$. Substituting Eq. (F12) for Eq. (F9), we obtain

$$
\begin{gathered}
h_{\nu \alpha}^{(m+1)}(\boldsymbol{\sigma})=-\sum_{\nu^{\prime}} \int \frac{d^{2} \boldsymbol{\sigma}^{\prime}}{(2 \pi)^{3}} \frac{\gamma_{\nu}\left(\boldsymbol{\sigma}^{\prime}, \mathbf{B}\right)}{\left|\mathbf{v}_{\nu}\left(\boldsymbol{\sigma}^{\prime}\right)\right|} \delta f_{\nu, \nu^{\prime}}\left(\boldsymbol{\sigma}, \boldsymbol{\sigma}^{\prime}\right) h_{\nu^{\prime} \alpha}^{(m)} \\
m=0,1,2 \ldots
\end{gathered}
$$

where $h_{\nu \alpha}^{(0)}=\delta_{\nu \alpha}$ and

$$
A_{\nu \alpha}=\delta_{\nu \alpha}+\sum_{\nu^{\prime}} \int \frac{d^{2} \boldsymbol{\sigma}^{\prime}}{(2 \pi)^{3}} \frac{\gamma_{\nu}\left(\boldsymbol{\sigma}^{\prime}, \mathbf{B}\right)}{\left|\mathbf{v}_{\nu}\left(\boldsymbol{\sigma}^{\prime}\right)\right|}\left(\bar{f}_{\nu, \nu^{\prime}}+\frac{e^{2}}{\epsilon_{0} \mathbf{q}^{2}}\right)\left(\delta_{\nu^{\prime} \alpha}+\sum_{m=1}^{\infty} h_{\nu^{\prime} \alpha}^{(m)}\left(\boldsymbol{\sigma}^{\prime}\right)\right)
$$

We can properly choose $\bar{f}_{\nu, \nu^{\prime}}$ such that

$$
\int \frac{d^{2} \boldsymbol{\sigma}}{(2 \pi)^{3}} \frac{\gamma_{\nu}(\boldsymbol{\sigma}, \mathbf{B})}{\left|\mathbf{v}_{\nu}(\boldsymbol{\sigma})\right|} \int \frac{d^{2} \boldsymbol{\sigma}^{\prime}}{(2 \pi)^{3}} \frac{\gamma_{\nu}\left(\boldsymbol{\sigma}^{\prime}, \mathbf{B}\right)}{\left|\mathbf{v}_{\nu^{\prime}}\left(\boldsymbol{\sigma}^{\prime}\right)\right|}\left(\delta f_{\nu, \nu^{\prime}}\left(\boldsymbol{\sigma}, \boldsymbol{\sigma}^{\prime}\right)+\sum_{\nu^{\prime \prime}} \int \frac{d^{2} \boldsymbol{\sigma}^{\prime \prime}}{(2 \pi)^{3}} \frac{\gamma_{\nu}\left(\boldsymbol{\sigma}^{\prime \prime}, \mathbf{B}\right)}{\left|\mathbf{v}_{\nu^{\prime \prime}}\left(\boldsymbol{\sigma}^{\prime \prime}\right)\right|} \delta f_{\nu, \nu^{\prime \prime}}\left(\boldsymbol{\sigma}, \boldsymbol{\sigma}^{\prime \prime}\right) \delta f_{\nu^{\prime \prime}, \nu^{\prime}}\left(\boldsymbol{\sigma}^{\prime \prime}, \boldsymbol{\sigma}^{\prime}\right)+\cdots\right)=0
$$

Then, due to Eqs. (F13) and (F15), there are

$$
\int \frac{d^{2} \boldsymbol{\sigma}}{(2 \pi)^{3}} \frac{\gamma_{\nu}(\boldsymbol{\sigma}, \mathbf{B})}{\left|\mathbf{v}_{\nu}(\boldsymbol{\sigma})\right|} h_{\nu \alpha}(\boldsymbol{\sigma})=\delta_{\nu \alpha} \beta_{\nu}(\mathbf{B})
$$

and

$$
A_{\nu \alpha}=\delta_{\nu \alpha}+\left(\bar{f}_{\nu, \alpha}+\frac{e^{2}}{\epsilon_{0} \mathbf{q}^{2}}\right) \beta_{\alpha}(\mathbf{B})
$$

To be specific, we can expand $\bar{f}_{\nu, \nu^{\prime}}$ that fulfills Eq. (F15) in orders of $f$ as 


$$
\bar{f}=\bar{f}^{(1)}+\bar{f}^{(2)}+\cdots,
$$

where

$$
\bar{f}_{\nu, \nu^{\prime}}^{(1)}=\frac{1}{\beta_{\nu}(\mathbf{B}) \beta_{\nu^{\prime}}(\mathbf{B})} \int \frac{d^{2} \boldsymbol{\sigma}}{(2 \pi)^{3}} \frac{\gamma_{\nu}(\boldsymbol{\sigma}, \mathbf{B})}{\left|\mathbf{v}_{\nu}(\boldsymbol{\sigma})\right|} \int \frac{d^{2} \boldsymbol{\sigma}^{\prime}}{(2 \pi)^{3}} \frac{\gamma_{\nu}\left(\boldsymbol{\sigma}^{\prime}, \mathbf{B}\right)}{\left|\mathbf{v}_{\nu^{\prime}}\left(\boldsymbol{\sigma}^{\prime}\right)\right|} f_{\nu, \nu^{\prime}}\left(\boldsymbol{\sigma}, \boldsymbol{\sigma}^{\prime}\right)
$$

and

$$
\begin{aligned}
\bar{f}_{\nu, \nu^{\prime}}^{(2)}= & \frac{1}{\beta_{\nu}(\mathbf{B}) \beta_{\nu^{\prime}}(\mathbf{B})} \int \frac{d^{2} \boldsymbol{\sigma}}{(2 \pi)^{3}} \frac{\gamma_{\nu}(\boldsymbol{\sigma}, \mathbf{B})}{\left|\mathbf{v}_{\nu}(\boldsymbol{\sigma})\right|} \int \frac{d^{2} \boldsymbol{\sigma}^{\prime}}{(2 \pi)^{3}} \frac{\gamma_{\nu}\left(\boldsymbol{\sigma}^{\prime}, \mathbf{B}\right)}{\left|\mathbf{v}_{\nu^{\prime}}\left(\boldsymbol{\sigma}^{\prime}\right)\right|} \\
& \times\left(\sum_{\nu^{\prime \prime}} \int \frac{d^{2} \boldsymbol{\sigma}^{\prime \prime}}{(2 \pi)^{3}} \frac{\gamma_{\nu}\left(\boldsymbol{\sigma}^{\prime \prime}, \mathbf{B}\right)}{\left|\mathbf{v}_{\nu^{\prime \prime}}\left(\boldsymbol{\sigma}^{\prime \prime}\right)\right|}\left(f_{\nu, \nu^{\prime \prime}}\left(\boldsymbol{\sigma}, \boldsymbol{\sigma}^{\prime \prime}\right)-f_{\nu, \nu^{\prime \prime}}^{(1)}\right)\left(f_{\nu^{\prime \prime}, \nu^{\prime}}\left(\boldsymbol{\sigma}^{\prime \prime}, \boldsymbol{\sigma}^{\prime}\right)-f_{\nu^{\prime \prime}, \nu^{\prime}}^{(1)}\right)\right) .
\end{aligned}
$$

\section{Dynamic equation}

Now we study the dynamic equation of the valley d.o.f. We first look at the scattering term. Since $d n_{\nu}(\boldsymbol{\sigma})=$ $\overline{\delta n}_{\nu}(\boldsymbol{\sigma})+\widetilde{\delta n}_{\nu}(\boldsymbol{\sigma})$ and $\mathcal{S}=\mathcal{S}^{(0)}+\mathcal{S}^{(1)}$, where $\mathcal{S}^{(0)}$ is contributed by intravalley scattering [Eq. (F6)] and $\mathcal{S}^{(1)}$ is contributed by intervalley scattering, the total scattering term decomposes into four terms

$$
\mathcal{S}\left[n_{\nu}(\boldsymbol{\sigma})\right]=\mathcal{S}^{(0)}\left[\overline{\delta n}_{\nu}(\boldsymbol{\sigma})\right]+\mathcal{S}^{(1)}\left[\overline{\delta n}_{\nu}(\boldsymbol{\sigma})\right]+\mathcal{S}^{(0)}\left[\widetilde{\delta n}_{\nu}(\boldsymbol{\sigma})\right]+\mathcal{S}^{(1)}\left[\widetilde{\delta n}_{\nu}(\boldsymbol{\sigma})\right]
$$

In the last subsection, we prove that $\mathcal{S}^{(0)}\left[\overline{\delta n}_{\nu}(\boldsymbol{\sigma})\right]=0$. Now we make a relaxation-time approximation for the other three terms

$$
\begin{gathered}
\mathcal{S}^{(1)}\left[\overline{\delta n}_{\nu}(\boldsymbol{\sigma})\right] \approx-\frac{\overline{\delta n}_{\nu}(\boldsymbol{\sigma})}{\tau_{v}}, \\
\mathcal{S}^{(0)}\left[\widetilde{\delta n}_{\nu}(\boldsymbol{\sigma})\right]+\mathcal{S}^{(1)}\left[\widetilde{\delta n}_{\nu}(\boldsymbol{\sigma})\right] \approx-\frac{\widetilde{\delta n_{\nu}}(\boldsymbol{\sigma})}{\tau_{0}} .
\end{gathered}
$$

In the chiral limit, we have $\mathcal{S}^{(0)} \gg \mathcal{S}^{(1)}$ and so $\tau_{0} \ll \tau_{v}$.

Following the derivation in Appendix A, we obtain the linearized Boltzmann equation with $\mathbf{k}$-dependent short-range interaction as

$$
\begin{aligned}
& \frac{\gamma_{\nu}(\boldsymbol{\sigma}, \mathbf{B})}{\left|\mathbf{v}_{\nu}(\boldsymbol{\sigma})\right|}\left[\left(\omega+\frac{i}{\tau_{v}}\right) \overline{\delta n}_{\nu}(\boldsymbol{\sigma})+\left(\omega+\frac{i}{\tau_{0}}\right) \widetilde{\delta n}_{\nu}(\boldsymbol{\sigma})\right] \\
& \quad=\left\{\mathbf{q} \cdot \hat{\mathbf{v}}_{\nu}(\boldsymbol{\sigma})-e \mathbf{q} \cdot \mathbf{B}\left[\hat{\mathbf{v}}_{\nu}(\boldsymbol{\sigma}) \cdot \boldsymbol{\Omega}_{\nu}(\boldsymbol{\sigma})\right]\right\}\left[\delta n_{\nu}(\boldsymbol{\sigma})+\Delta_{\nu}(\boldsymbol{\sigma})\right]-i e\left[\hat{\mathbf{v}}_{\nu}(\boldsymbol{\sigma}) \times \mathbf{B}\right] \cdot \partial_{\mathbf{k}}\left[\delta n_{\nu}(\boldsymbol{\sigma})+\Delta_{\nu}(\boldsymbol{\sigma})\right],
\end{aligned}
$$

where $\Delta_{\nu}(\boldsymbol{\sigma})$ is defined in Eq. (F2). To zeroth order of $\tau_{0}$, we have

$$
\delta n_{\nu}(\boldsymbol{\sigma})=\overline{\delta n}_{\nu}(\boldsymbol{\sigma})=\sum_{\alpha} c_{\alpha} h_{\nu \alpha}(\boldsymbol{\sigma})
$$

where $h_{\nu \alpha}(\boldsymbol{\sigma})$ are the bases introduced in the last subsection. Because of Eq. (F7), $\delta n_{\nu}(\boldsymbol{\sigma})+\Delta_{\nu}(\boldsymbol{\sigma})$ is a constant $a_{\nu}$, and due to Eqs. (F10) and (F17), Eq. (F24) can be written as

$$
\frac{\gamma_{\nu}(\boldsymbol{\sigma}, \mathbf{B})}{\left|\mathbf{v}_{\nu}(\boldsymbol{\sigma})\right|}\left(\omega+\frac{i}{\tau_{v}}\right) \overline{\delta n}_{\nu}(\boldsymbol{\sigma})=\left\{\mathbf{q} \cdot \hat{\mathbf{v}}_{\nu}(\boldsymbol{\sigma})-e \mathbf{q} \cdot \mathbf{B}\left[\hat{\mathbf{v}}_{\nu}(\boldsymbol{\sigma}) \cdot \mathbf{\Omega}_{\nu}(\boldsymbol{\sigma})\right]\right\}\left[c_{\nu}+\sum_{\nu^{\prime}}\left(\bar{f}_{\nu, \nu^{\prime}}+\frac{e^{2}}{\epsilon_{0} \mathbf{q}^{2}}\right) \beta_{\alpha}(\mathbf{B}) c_{\nu^{\prime}}\right] .
$$

Integrating $\boldsymbol{\sigma}$ on both sides of this equation and applying Eq. (F16), we obtain 


$$
\left(\omega+\frac{i}{\tau_{v}}\right) \beta_{\nu}(\mathbf{B}) c_{\nu}=\chi_{\nu} \frac{e(\mathbf{q} \cdot \mathbf{B})}{4 \pi^{2}}\left[c_{\nu}+\sum_{\nu^{\prime}}\left(\bar{f}_{\nu, \nu^{\prime}}+\frac{e^{2}}{\epsilon_{0} \mathbf{q}^{2}}\right) \beta_{\nu^{\prime}}(\mathbf{B}) c_{\nu^{\prime}}\right] .
$$

We introduce the variable $\eta_{\nu}=\beta_{\nu}(\mathbf{B}) c_{\nu}$, and then we obtain

$$
\left(\omega+\frac{i}{\tau_{v}}\right) \chi_{\nu} \eta_{\nu}=\frac{e(\mathbf{q} \cdot \mathbf{B})}{4 \pi^{2} \beta_{\nu}(\mathbf{B})} \eta_{\nu}+\frac{e(\mathbf{q} \cdot \mathbf{B})}{4 \pi^{2}} \sum_{\nu^{\prime}}\left(\bar{f}_{\nu, \nu^{\prime}}+\frac{e^{2}}{\epsilon_{0} \mathbf{q}^{2}}\right) \eta_{\nu^{\prime}}
$$

which is of the same form as Eq. (B6).

\section{APPENDIX G: THERMODYNAMIC PROPERTY OF CHIRAL ZERO SOUND}

We treat the CZS modes as bosonic quasiparticle excitations. For each branch of CZS modes $\omega_{n}(\mathbf{q})$, we assign a distribution function $g_{n}(\mathbf{q}, \mathbf{r}, t)$, and in equilibrium it is just the Bose-Einstein distribution, i.e.,

$$
g_{n}^{(0)}(\mathbf{q}, \mathbf{r}, t)=\frac{1}{\exp \left(\frac{\omega_{n}(\mathbf{q})}{k_{B} T}\right)-1},
$$

where $k_{B}$ is the Boltzmann constant and $T$ is the temperature. Here we drop the "CZS" subscript for brevity. In the following, we assume the magnetic field is applied along the $z$ direction, so the dispersion is $\omega_{n}(\mathbf{q})=c_{n} q_{z}$.

First let us calculate the specific heat per unit volume

$$
\begin{aligned}
\kappa(T)= & \frac{\partial}{\partial T} \sum_{n} \int_{|\mathbf{q}|<\Lambda} \frac{d^{3} \mathbf{q}}{(2 \pi)^{3}} \frac{\left|c_{n} q_{z}\right|}{\exp \left(\frac{\left|c_{n} q_{z}\right|}{k_{B} T}\right)-1} \\
= & \sum_{n} k_{B} \int_{-\Lambda}^{\Lambda} \frac{d q_{z}}{2 \pi} \frac{\Lambda^{2}-q_{z}^{2}}{4 \pi}\left(\frac{c_{n} q_{z}}{k_{B} T}\right)^{2} \frac{1}{4 \sinh ^{2} \frac{c_{n} q_{z}}{2 k_{B} T}} \\
= & \sum_{n} \kappa_{n}(T), \\
\kappa_{n}(T)= & k_{B} \Lambda^{3}\left(\frac{k_{B} T}{c_{n} \Lambda}\right)^{3} \int_{-c_{n} \Lambda /\left(k_{B} T\right)}^{c_{n} \Lambda /\left(k_{B} T\right)} \frac{d x}{2 \pi} \\
& \times \frac{\left[c_{n} \Lambda /\left(k_{B} T\right)\right]^{2}-x^{2}}{4 \pi} \frac{x^{2}}{4 \sinh ^{2} \frac{x}{2}},
\end{aligned}
$$

where $\Lambda \sim 1 / a_{0}$ is the cutoff of $q, a_{0}$ is a lattice constant, and $\kappa_{n}$ is the specific heat contributed by the $n$th branch of the CZS. In the two limits $c_{n} \Lambda \gg k_{B} T$ and $c_{n} \Lambda \ll k_{B} T$, we have

$$
\kappa_{n}(T)= \begin{cases}k_{B} \Lambda^{3} \frac{k_{B} T}{12 c_{n} \Lambda}, & c_{n} \Lambda \gg k_{B} T, \\ k_{B} \Lambda^{3} \frac{1}{6 \pi^{2}}, & c_{n} \Lambda \ll k_{B} T .\end{cases}
$$

Now let us calculate the thermal conductivity. For an inhomogeneous system, the distribution function satisfies the Boltzmann equation

$$
\partial_{t} g_{n}(\mathbf{q}, \mathbf{r}, t)=-c_{n} \partial_{z} g_{n}(\mathbf{q}, \mathbf{r}, t)-\frac{g_{n}(\mathbf{q}, \mathbf{r}, t)-g_{n}^{(0)}(\mathbf{q}, \mathbf{r}, t)}{\tau_{s}(T)},
$$

where $\tau_{s}(T)$ is the relaxation time for the CZS excitations. At low temperature, the relaxation should be proportional to $\tau_{v}$. In the presence of a temperature gradient, the firstorder stationary solution reads

$$
\begin{aligned}
\delta g_{n}(\mathbf{q}, \mathbf{r})= & g_{n}(\mathbf{q}, \mathbf{r})-g_{n}^{(0)}(\mathbf{q}, \mathbf{r}) \approx-\tau_{s}(T)\left(c_{n} \partial_{z} T\right) \\
& \times \frac{\omega_{n}(\mathbf{q})}{k_{B} T^{2}} \frac{1}{4 \sinh ^{2} \frac{\omega_{n}(q)}{2 k_{B} T}} .
\end{aligned}
$$

The thermal current is given by

$$
\begin{aligned}
j_{z}^{\text {th }} & =\sum_{n} \int_{|\mathbf{q}|<\Lambda} \frac{d^{3} \mathbf{q}}{(2 \pi)^{3}} \omega_{n}(\mathbf{q}) c_{n} \delta g_{n}(\mathbf{q}, \mathbf{r}) \\
& =-\tau_{s}(T) \sum_{n} \int_{|\mathbf{q}|<\Lambda} \frac{d^{3} \mathbf{q}}{(2 \pi)^{3}} c_{n}\left(c_{n} \partial_{z} T\right) \frac{\omega_{n}^{2}(\mathbf{q})}{k_{B} T^{2}} \frac{1}{4 \sinh ^{2} \frac{\omega_{n}(\mathbf{q})}{2 k_{B} T}} .
\end{aligned}
$$

Therefore, the thermal conductivity is

$$
\sigma_{i, j}^{\mathrm{th}}=\delta_{i, z} \delta_{j, z} \tau_{\mathrm{s}}(T) \sum_{n} c_{n}^{2} \kappa_{n}(T)
$$

\section{APPENDIX H: STRONG MAGNETIC FIELD AND FINITE TEMPERATURE}

The above derivations are based on Boltzmann's equation, which is valid only if $\omega_{B} \tau_{0} \ll 1, \omega_{B} \ll \mu$. Thus, it is still unknown whether the CP and CZS modes exist in the case $\omega_{B} \tau_{0} \gtrsim 1, \omega_{B} \ll \mu$. Here we refer to this case as the strong field case. In this case, the Landau levels are formed, and there are many Landau levels under the chemical potential. Therefore, the system should be described by distribution functions on the Landau levels. Here we expand this distribution function as an equilibrium part and a small deviation from equilibrium

$$
\begin{aligned}
n_{\nu}(k, \alpha, t)= & n_{T}\left[\epsilon_{\nu}^{0}(k, \alpha)-\mu\right] \\
& +\delta_{T}\left[\epsilon_{\nu}^{0}(k, \alpha)-\mu\right] \delta n_{\nu}(\alpha) e^{i(\mathbf{q} \cdot \mathbf{r}-\omega t)} .
\end{aligned}
$$


Here, $k$ is the momentum along the magnetic field, $\alpha$ is the Landau-level index, $\epsilon_{\nu}^{0}(k, \alpha)$ are the Landau levels, $n_{0}\left[\epsilon_{\nu}^{0}(k, \alpha)-\mu\right]=\left\langle\psi_{k \alpha}^{\dagger} \psi_{k \alpha}\right\rangle$ is the occupation number in equilibrium, and $\delta_{0}(\epsilon)=-\partial_{\epsilon} n_{0}(\epsilon)$. We assume the Landau levels as [57]

$$
\epsilon_{\nu}^{0}(k, \alpha)= \begin{cases}u k+v \sqrt{k^{2}+2 e B \alpha,} & \alpha>0, \\ u k+\chi v k, & \alpha=0, \\ u k-v \sqrt{k^{2}+2 e B|\alpha|,} & \alpha<0,\end{cases}
$$

where $|u|<|v|$ such that the WP is type I [11]. In the presence of scattering Eq. (E1), we can write the spectrum function as [58]

$A\left[\epsilon_{\nu}^{0}(\mathbf{k}, \alpha), \omega\right]=\frac{1}{\pi} \frac{1 /\left(2 \tau_{0}\right)}{\left[\epsilon_{\nu}^{0}(\mathbf{k}, \alpha)-\mu-\omega\right]^{2}+1 /\left(2 \tau_{0}\right)^{2}}$,

where $\tau_{0}$ is the quasiparticle lifetime [Eq. (E5)]. Therefore, the occupation number is given by

$$
n_{T}(\epsilon)=\int d \omega \frac{1}{1+\exp \frac{\omega}{k_{B} T}} A(\epsilon, \omega)
$$

and its derivative is given by

$$
\delta_{T}(\epsilon)=\int d \omega \frac{1 /\left(2 k_{B} T\right)}{1+\cosh \frac{\omega}{k_{B} T}} A(\epsilon, \omega) .
$$

Similar to the weak field case, we decompose $\delta n_{\nu}(\alpha)$ as a valley degree

$$
\overline{\delta n}_{\nu}=\frac{1}{\beta_{\nu}(B)} \frac{e B}{2 \pi} \int \frac{d k}{2 \pi} \sum_{\alpha} \delta_{T}\left[\epsilon_{\nu}^{0}(k, \alpha)-\mu\right] \delta n_{\nu}(\alpha)
$$

and a Fermi-surface degree

$$
\widetilde{\delta n_{\nu}}(\alpha)=\delta n_{\nu}(\alpha)-\overline{\delta n}_{\nu},
$$

where

$$
\beta_{\nu}(B)=\frac{e B}{2 \pi} \int \frac{d k}{2 \pi} \sum_{\alpha} \delta_{T}\left[\epsilon_{\nu}^{0}(k, \alpha)-\mu\right]
$$

is the compressibility at finite temperature. Here we assume that $e B>0$. Then, the kinetic equation of collective modes can be written as

$$
\begin{aligned}
& \delta_{T}\left[\epsilon_{\nu}^{0}(k, \alpha)-\mu\right]\left[\left(\omega+\frac{i}{\tau_{v}}\right) \overline{\delta n}_{\nu}+\left(\omega+\frac{i}{\tau_{0}}\right) \widetilde{\delta n}_{\nu}(\alpha)\right] \\
& =\delta_{T}\left[\epsilon_{\nu}^{0}(k, \alpha)-\mu\right] \mathbf{q} \cdot \mathbf{v}_{\nu}(\alpha) \\
& \quad \times\left[\delta n_{\nu}(\alpha)+\sum_{\nu^{\prime}}\left(f_{\nu, \nu^{\prime}}+\frac{e^{2}}{\epsilon_{0} \mathbf{q}^{2}}\right) \beta_{\nu^{\prime}}(B) \overline{\delta n}_{\nu^{\prime}}\right] .
\end{aligned}
$$

We define the disequilibrium quasiparticle number in the $\nu$ th valley as $\eta_{\nu}=\beta_{\nu}(B) \overline{\delta n}_{\nu}$. Then, to zeroth order of $\tau_{0}$, integrating $k$ and summing over $\alpha$, we get

$$
\begin{aligned}
\left(\omega+\frac{i}{\tau_{v}}\right) \chi_{\nu} \eta_{\nu}= & \frac{e(\mathbf{q} \cdot \mathbf{B})}{4 \pi^{2} \beta_{\nu}(\mathbf{B})} \eta_{\nu}+\frac{e(\mathbf{q} \cdot \mathbf{B})}{4 \pi^{2}} \\
& \times \sum_{\nu^{\prime}}\left(f_{\nu, \nu^{\prime}}+\frac{e^{2}}{\epsilon_{0} \mathbf{q}^{2}}\right) \eta_{\nu^{\prime}},
\end{aligned}
$$

which has the exact form as Eq. (B6). It should be noticed that due to Eq. (H2), only the zeroth Landau level contributes to the integral on the rhs. One can easily verify that the leading-order effect of $\tau_{v}$ is introducing an effective damping rate, and the stable condition for the CZS and CP modes is still given by Eqs. (E15) and (E17), respectively.

\section{APPENDIX I: QUANTUM OSCILLATION IN COMPRESSIBILITY}

After the Landau levels are formed, the compressibility at finite temperature is given by

$\beta_{\nu}(B)=\sum_{\alpha} F(\alpha), \quad F(\alpha)=\frac{e B}{2 \pi} \int \frac{d k}{2 \pi} \delta_{0}\left[\epsilon_{\nu}^{0}(k, \alpha)-\mu\right]$.

Here we assume $\mu>0$. Using the Poisson equation, we get [42]

$$
\begin{aligned}
\beta_{\nu}(B)= & F(0)-\frac{1}{2} F\left(0^{+}\right)+\frac{1}{2} F\left(0^{+}\right)+\sum_{\alpha=1}^{\infty} F(\alpha) \\
= & F(0)-\frac{1}{2} F\left(0^{+}\right)+\int_{0}^{\infty} d \alpha F(\alpha) \\
& +2 \Re \sum_{l=1}^{\infty} \int_{0}^{\infty} d \alpha F(\alpha) e^{2 \pi i l \alpha} .
\end{aligned}
$$

We define

$$
\beta_{\nu}^{(0)}(B)=F(0)-\frac{1}{2} F\left(0^{+}\right)+\int_{0}^{\infty} d \alpha F(\alpha)
$$

as the nonoscillating component and 


$$
\beta_{\nu}^{(l)}(B)=2 \Re \int_{0}^{\infty} d \alpha F(\alpha) e^{2 \pi i l \alpha}, \quad l \geq 1
$$

as the oscillating components. $\beta_{\nu}^{(0)}(B)$ is just the compressibility in the weak field limit [Eq. (B4)]. Now let us calculate $\beta_{\nu}^{(l)}(B)$. We use the relation

$$
\alpha=\frac{S_{\nu}(\epsilon, k)}{2 \pi e B}-\phi_{\nu}(\epsilon, k)
$$

where $S_{\nu}$ is the area enclosed by the fixed-energy circle in the $k$ plane. $\phi(\epsilon, k)$ includes the Maslov index plus the Berry phase. For linear isotropic WPs, we always have $\phi_{\nu}(\epsilon, \mathbf{k})=0$, and so in the following, we omit $\phi_{\nu}(\epsilon, k)$. Expanding $S_{\nu}(\epsilon, k)$ as

$$
S_{\nu}(\epsilon, k) \approx S_{\mathrm{ex}, \nu}(\epsilon)+\left.\frac{1}{2} \frac{\partial^{2} S_{\nu}}{\partial k^{2}}\right|_{\mathrm{ex}}\left(k-k_{\mathrm{ex}}\right)^{2}
$$

we then have

$$
\begin{aligned}
\beta_{\nu}^{(l)}(B) & =\mathfrak{R} \frac{e B}{\pi} \int_{0}^{\infty} d \alpha \int \frac{d k}{2 \pi} \exp (i 2 \pi l \alpha) \delta_{T}\left[\epsilon_{\nu}^{0}(k, \alpha)-\mu\right] \\
& =\Re \frac{1}{2 \pi^{2}} \int d \epsilon \int \frac{d k}{2 \pi} \frac{d S_{\mathrm{ex}, \nu}(\epsilon)}{d \epsilon} \exp \left(i 2 \pi l \frac{S_{\mathrm{ex}, \nu}(\epsilon)+\left.\frac{1}{2} \frac{\partial^{2} S_{\nu}}{\partial k^{2}}\right|_{\mathrm{ex}}\left(k-k_{\mathrm{ex}}\right)^{2}}{2 \pi e B}\right) \delta_{T}(\epsilon-\mu) .
\end{aligned}
$$

Applying the Gaussian integral formula $\int_{-\infty}^{\infty} \exp \left[(i / 2) a x^{2}\right] d x=[(2 \pi i) / a]^{\frac{1}{2}}$, we get

$$
\beta_{\nu}^{(l)}(B)=\Re \frac{1}{4 \pi^{3}} \int d \epsilon\left(l\left|\frac{\partial^{2} S_{\nu}}{\partial k^{2}}\right|_{\mathrm{ex}} \frac{1}{2 \pi e B}\right)^{-\frac{1}{2}} \frac{d S_{\mathrm{ex}, \nu}(\epsilon)}{d \epsilon} \exp \left(i 2 \pi l \frac{S_{\mathrm{ex}, \nu}(\epsilon)}{2 \pi e B}-i \frac{\pi}{4}\right) \delta_{T}(\epsilon-\mu),
$$

where we assume $\left[\left(\partial^{2} S_{\nu}\right) /\left(\partial k^{2}\right)\right]<0$. Substituting Eq. (H5) into the above equation, we get

$$
\begin{aligned}
\beta_{\nu}^{(l)}(B)= & \Re \frac{1}{4 \pi^{3}} \int d \epsilon \int d \omega\left(l\left|\frac{\partial^{2} S_{\nu}}{\partial k^{2}}\right|_{\mathrm{ex}} \frac{1}{2 \pi e B}\right)^{-\frac{1}{2}} \frac{d S_{\mathrm{ex}, \nu}(\epsilon)}{d \epsilon} \exp \left(i 2 \pi l \frac{S_{\mathrm{ex}, \nu}(\epsilon)}{2 \pi e B}-i \frac{\pi}{4}\right) \\
& \times \frac{1 /\left(2 k_{B} T\right)}{1+\cosh \left(\frac{\omega}{k_{B} T}\right)} \frac{1}{\pi} \frac{1 / 2 \tau_{0}}{(\epsilon-\mu-\omega)^{2}+1 /\left(2 \tau_{0}\right)^{2}} .
\end{aligned}
$$

Using the contour integral in the upper half plane of $\epsilon$, we get

$$
\begin{aligned}
\beta_{\nu}^{(l)}(B)= & \left.\Re \frac{1}{4 \pi^{3}} \int d \omega\left(l\left|\frac{\partial^{2} S_{\nu}}{\partial k^{2}}\right|_{\mathrm{ex}} \frac{1}{2 \pi e B}\right)^{-\frac{1}{2}} \frac{d S_{\mathrm{ex}, \nu}(\epsilon)}{d \epsilon}\right|_{\epsilon=\mu+\omega+i /\left(2 \tau_{0}\right)} \exp \left(i 2 \pi l \frac{S_{\mathrm{ex}, \nu}\left(\mu+\omega+\frac{i}{2 \tau_{0}}\right)}{2 \pi e B}-i \frac{\pi}{4}\right) \\
& \times \frac{1 /\left(2 k_{B} T\right)}{1+\cosh \frac{\omega}{k_{B} T}} .
\end{aligned}
$$

We approximate $S_{\mathrm{ex}, \nu}\left\{\mu+\omega+\left[i /\left(2 \tau_{0}\right)\right]\right\}$ as $S_{\mathrm{ex}, \nu}(\mu)+\left\{\left[d S_{\mathrm{ex}, \nu}(\mu)\right] /(d \mu)\right\}\left\{\omega+\left[i /\left(2 \tau_{0}\right)\right]\right\}$, then we have

$$
\begin{aligned}
\beta_{\nu}^{(l)}(B) \approx & \Re \frac{1}{8 \pi^{3}} \frac{d S_{\mathrm{ex}, \nu}(\mu)}{d \mu}\left(l\left|\frac{\partial^{2} S_{\nu}}{\partial k^{2}}\right|_{\mathrm{ex}} \frac{1}{2 \pi e B}\right)^{-\frac{1}{2}} \exp \left(-\frac{d S_{\mathrm{ex}, \nu}(\mu)}{d \mu} \frac{l}{2 e B \tau_{0}}\right) \exp \left(i 2 \pi l \frac{S_{\mathrm{ex}, \nu}(\mu)}{2 \pi e B}-i \frac{\pi}{4}\right) \\
& \times \int d \omega \frac{1}{k_{B} T} \frac{\exp \left(i \frac{d S_{\mathrm{ex}, \nu}(\mu)}{d \mu} \frac{2 \omega l}{2 e B}\right)}{1+\cosh \frac{\omega}{k_{B} T}}
\end{aligned}
$$

Now we need to calculate the integral on the second line of the above equation. We denote this integral as $I$. We choose the contour $-\infty \rightarrow \infty \rightarrow \infty+i 2 \pi \rightarrow-\infty+i 2 \pi \rightarrow-\infty$, and then we have

$$
\left[1-\exp \left(-2 \pi \frac{d S_{\mathrm{ex}, \nu}(\mu)}{d \mu} \frac{k_{B} T l}{e B}\right)\right] I=4 \pi \frac{d S_{\mathrm{ex}, \nu}(\mu)}{d \mu} \frac{k_{B} T l}{e B} \exp \left(-\pi \frac{d S_{\mathrm{ex}, \nu}(\mu)}{d \mu} \frac{k_{B} T l}{e B}\right),
$$


and thus,

$$
I=\frac{2}{\operatorname{sinch}\left(\frac{d S_{\text {ex, }, ~}(\mu)}{d \mu} \frac{\pi k_{B} T l}{e B}\right)},
$$

where $\operatorname{sinch}(x)=\left(e^{x}-e^{-x}\right) /(2 x)$. Therefore, we get

$$
\beta_{\nu}^{(l)}(B) \approx \frac{1}{4 \pi^{3}} \frac{d S_{\mathrm{ex}, \nu}(\mu)}{d \mu}\left(l\left|\frac{\partial^{2} S_{\nu}}{\partial k^{2}}\right|_{\mathrm{ex}, \mu} \frac{1}{2 \pi e B}\right)^{-\frac{1}{2}} \frac{\exp \left(-\frac{d S_{\mathrm{ex}, \nu}(\mu)}{d \mu} \frac{l}{2 e B \tau_{0}}\right)}{\operatorname{sinch}\left(\frac{d S_{\mathrm{ex}, \nu}(\mu)}{d \mu} \frac{\pi k_{B} T l}{e B}\right)} \cos \left(2 \pi l \frac{S_{\mathrm{ex}, \nu}(\mu)}{2 \pi e B}-\frac{\pi}{4}\right) .
$$

For an isotropic Fermi surface, where $\epsilon=v_{F}|\mathbf{k}|$ and $S(\epsilon, k)=\pi\left[\left(\epsilon / v_{F}\right)^{2}-k^{2}\right]$, the first-order oscillation is given by

$\beta_{\nu}^{(1)}(B) \approx \frac{1}{2 \pi^{2}} \frac{\mu \omega_{B}}{v_{F}^{3}} \frac{\exp \left(-2 \pi \frac{\mu}{\omega_{B}^{2} 2 \tau_{0}}\right)}{\operatorname{sinch}\left(2 \pi^{2} \frac{\mu k_{B} T}{\omega_{B}^{2}}\right)} \cos \left(\frac{S_{\mathrm{ex}, \nu}(\mu)}{e B}-\frac{\pi}{4}\right)$

and

$\frac{\beta_{\nu}^{(1)}(B)}{\beta_{\nu}^{(0)}(B)} \approx \frac{\omega_{B}}{\mu} \frac{\exp \left(-\pi \frac{\mu}{\omega_{B}^{2} \tau_{0}}\right)}{\operatorname{sinch}\left(2 \pi^{2} \frac{\mu k_{B} T}{\omega_{B}^{2}}\right)} \cos \left(\frac{S_{\mathrm{ex}, \nu}(\mu)}{e B}-\frac{\pi}{4}\right)$.

[1] H. B. Nielsen and M. Ninomiya, The Adler-Bell-Jackiw Anomaly and Weyl Fermions in a Crystal, Phys. Lett. 130B, 389 (1983).

[2] S. Murakami, Phase Transition between the Quantum Spin Hall and Insulator Phases in 3D: Emergence of a Topological Gapless Phase, New J. Phys. 9, 356 (2007).

[3] X. Wan, A. M. Turner, A. Vishwanath, and S. Y. Savrasov, Topological Semimetal and Fermi-Arc Surface States in the Electronic Structure of Pyrochlore Iridates, Phys. Rev. B 83, 205101 (2011).

[4] A. A. Burkov and L. Balents, Weyl Semimetal in a Topological Insulator Multilayer, Phys. Rev. Lett. 107, 127205 (2011).

[5] H. Weng, C. Fang, Z. Fang, B. A. Bernevig, and X. Dai, Weyl Semimetal Phase in Noncentrosymmetric Transition-Metal Monophosphides, Phys. Rev. X 5, 011029 (2015).

[6] B.-J. Yang and N. Nagaosa, Classification of Stable ThreeDimensional Dirac Semimetals with Nontrivial Topology, Nat. Commun. 5, 4898 (2014).

[7] A. A. Burkov, M. D. Hook, and L. Balents, Topological Nodal Semimetals, Phys. Rev. B 84, 235126 (2011).

[8] Y. Kim, B. J. Wieder, C. L. Kane, and A. M. Rappe, Dirac Line Nodes in Inversion-Symmetric Crystals, Phys. Rev. Lett. 115, 036806 (2015).
[9] R. Yu, H. Weng, Z. Fang, X. Dai, and X. Hu, Topological Node-Line Semimetal and Dirac Semimetal State in Antiperovskite $\mathrm{Cu}_{3} \mathrm{PdN}$, Phys. Rev. Lett. 115, 036807 (2015).

[10] C. Fang, Y. Chen, H.-Y. Kee, and L. Fu, Topological Nodal Line Semimetals with and without Spin-Orbital Coupling, Phys. Rev. B 92, 081201(R) (2015).

[11] A. A. Soluyanov, D. Gresch, Z. Wang, Q. S. Wu, M. Troyer, X. Dai, and B.A. Bernevig, Type-II Weyl Semimetals, Nature (London) 527, 495 (2015).

[12] H. Weyl, Elektron und Gravitation. I, Z. Phys. 56, 330 (1929).

[13] B. Q. Lv, N. Xu, H. M. Weng, J. Z. Ma, P. Richard, X. C. Huang, L. X. Zhao, G. F. Chen, C. E. Matt, F. Bisti, V. N. Strocov, J. Mesot, Z. Fang, X. Dai, T. Qian, M. Shi, and H. Ding, Observation of Weyl Nodes in TaAs, Nat. Phys. 11, 724 (2015).

[14] B. Q. Lv, S. Muff, T. Qian, Z. D. Song, S. M. Nie, N. Xu, P. Richard, C. E. Matt, N. C. Plumb, L. X. Zhao, G. F. Chen, Z. Fang, X. Dai, J. H. Dil, J. Mesot, M. Shi, H. M. Weng, and H. Ding, Observation of Fermi-Arc Spin Texture in TaAs, Phys. Rev. Lett. 115, 217601 (2015).

[15] S.-Y. Xu et al., Discovery of a Weyl Fermion Semimetal and Topological Fermi Arcs, Science 349, 613 (2015).

[16] S.-Y. Xu et al., Discovery of a Weyl Fermion State with Fermi Arcs in Niobium Arsenide, Nat. Phys. 11, 748 (2015).

[17] S.-M. Huang, S.-Y. Xu, I. Belopolski, C.-C. Lee, G. Chang, B. Wang, N. Alidoust, G. Bian, M. Neupane, C. Zhang, S. Jia, A. Bansil, H. Lin, and M. Z. Hasan, A Weyl Fermion Semimetal with Surface Fermi Arcs in the Transition Metal Monopnictide TaAs Class, Nat. Commun. 6, 7373 (2015).

[18] G. M. Andolina, F. M. D. Pellegrino, F. H. L. Koppens, and M. Polini, Quantum Nonlocal Theory of Topological Fermi Arc Plasmons in Weyl Semimetals, Phys. Rev. B 97, 125431 (2018).

[19] J. Xiong, S. K. Kushwaha, T. Liang, J. W. Krizan, M. Hirschberger, W. Wang, R. J. Cava, and N. P. Ong, Evidence for the Chiral Anomaly in the Dirac Semimetal $\mathrm{Na}_{3} \mathrm{Bi}$, Science 350, 413 (2015).

[20] X. Huang, L. Zhao, Y. Long, P. Wang, D. Chen, Z. Yang, H. Liang, M. Xue, H. Weng, Z. Fang, X. Dai, and G. Chen, Observation of the Chiral-Anomaly-Induced Negative Magnetoresistance in $3 D$ Weyl Semimetal TaAs, Phys. Rev. X 5, 031023 (2015).

[21] C.-Z. Li, L.-X. Wang, H. Liu, J. Wang, Z.-M. Liao, and D.-P. Yu, Giant Negative Magnetoresistance Induced by the Chiral Anomaly in Individual $\mathrm{Cd}_{3} \mathrm{As}_{2}$ Nanowires, Nat. Commun. 6, 10137 (2015). 
[22] C.-L. Zhang et al., Signatures of the Adler-Bell-Jackiw Chiral Anomaly in a Weyl fermion Semimetal, Nat. Commun. 7, 10735 (2016).

[23] H. Li, H. He, H.-Z. Lu, H. Zhang, H. Liu, R. Ma, Z. Fan, S.-Q. Shen, and J. Wang, Negative Magnetoresistance in Dirac Semimetal $\mathrm{Cd}_{3} \mathrm{As}_{2}$, Nat. Commun. 7, 10301 (2016).

[24] D. Son and N. Yamamoto, Berry Curvature, Triangle Anomalies, and the Chiral Magnetic Effect in Fermi Liquids, Phys. Rev. Lett. 109, 181602 (2012).

[25] M. A. Stephanov and Y. Yin, Chiral Kinetic Theory, Phys. Rev. Lett. 109, 162001 (2012).

[26] A. A. Burkov, Chiral Anomaly and Diffusive Magnetotransport in Weyl Metals, Phys. Rev. Lett. 113, 247203 (2014).

[27] E. V. Gorbar, V. A. Miransky, and I. A. Shovkovy, Chiral Anomaly, Dimensional Reduction, and Magnetoresistivity of Weyl and Dirac Semimetals, Phys. Rev. B 89, 085126 (2014).

[28] C.-X. Liu, P. Ye, and X.-L. Qi, Chiral Gauge Field and Axial Anomaly in a Weyl Semimetal, Phys. Rev. B 87, 235306 (2013).

[29] M. Lv and S.-C. Zhang, Dielectric Function, Friedel Oscillation and Plasmons in Weyl Semimetals, Int. J. Mod. Phys. B 27, 1350177 (2013).

[30] I. Panfilov, A. A. Burkov, and D. A. Pesin, Density Response in Weyl Metals, Phys. Rev. B 89, 245103 (2014).

[31] M. Stephanov, H.-U. Yee, and Y. Yin, Collective Modes of Chiral Kinetic Theory in a Magnetic Field, Phys. Rev. D 91, 125014 (2015).

[32] J. Zhou, H.-R. Chang, and D. Xiao, Plasmon Mode as a Detection of the Chiral Anomaly in Weyl Semimetals, Phys. Rev. B 91, 035114 (2015).

[33] F. M. D. Pellegrino, M. I. Katsnelson, and M. Polini, Helicons in Weyl Semimetals, Phys. Rev. B 92, 201407(R) (2015).

[34] Z. Song, J. Zhao, Z. Fang, and X. Dai, Detecting the Chiral Magnetic Effect by Lattice Dynamics in Weyl Semimetals, Phys. Rev. B 94, 214306 (2016).

[35] P. Rinkel, P. L. S. Lopes, and I. Garate, Signatures of the Chiral Anomaly in Phonon Dynamics, Phys. Rev. Lett. 119, 107401 (2017).

[36] E. V. Gorbar, V. A. Miransky, I. A. Shovkovy, and P. O. Sukhachov, Consistent Chiral Kinetic Theory in Weyl Materials: Chiral Magnetic Plasmons, Phys. Rev. Lett. 118, 127601 (2017).

[37] L. D. Landau, The Theory of a Fermi Liquid, Sov. Phys. JETP 3, 920 (1957).

[38] A. A. Abrikosov and I. M. Khalatnikov, The Theory of a Fermi Liquid (The Properties of Liquid ${ }^{3} \mathrm{He}$ at Low Temperatures), Rep. Prog. Phys. 22, 329 (1959).

[39] A. J. Leggett, A Theoretical Description of the New Phases of Liquid ${ }^{3} \mathrm{He}$, Rev. Mod. Phys. 47, 331 (1975).
[40] G. E. Volovik, The Universe in a Helium Droplet, International Series of Monographs on Physics Vol. 117 (Clarendon Press, Oxford, 2003).

[41] A. A. Abrikosov, L. P. Gorkov, and I. E. Dzialoshinskii, Methods of Quantum Field Theory in Statistical Physics (Dover Publications, New York, 1975).

[42] E. M. Lifshitz and L. P. Pitaevskii, Statistical Physics: Theory of the Condensed State (Elsevier, New York, 2013).

[43] D. Pines and P. Nozieres, Theory of Quantum Liquids, Volume I: Normal Fermi Liquids (Westview Press, Boulder, Colorado1994), Chap. 3, pp. 143-201.

[44] S.-K. Yip and T.-L. Ho, Zero Sound Modes of Dilute Fermi Gases with Arbitrary Spin, Phys. Rev. A 59, 4653 (1999).

[45] K. Fukushima, D. E. Kharzeev, and H. J. Warringa, Chiral Magnetic Effect, Phys. Rev. D 78, 074033 (2008).

[46] A. A. Zyuzin and A. A. Burkov, Topological Response in Weyl Semimetals and the Chiral Anomaly, Phys. Rev. B 86, 115133 (2012).

[47] M. M. Vazifeh and M. Franz, Electromagnetic Response of Weyl Semimetals, Phys. Rev. Lett. 111, 027201 (2013).

[48] Y. Chen, Si Wu, and A. A. Burkov, Axion Response in Weyl Semimetals, Phys. Rev. B 88, 125105 (2013).

[49] D. Culcer, Y. Yao, and Q. Niu, Coherent Wave-Packet Evolution in Coupled Bands, Phys. Rev. B 72, 085110 (2005).

[50] D. Xiao, M.-C. Chang, and Q. Niu, Berry Phase Effects on Electronic Properties, Rev. Mod. Phys. 82, 1959 (2010).

[51] D. Xiao, J. Shi, and Q. Niu, Berry Phase Correction to Electron Density of States in Solids, Phys. Rev. Lett. 95, 137204 (2005).

[52] V. P. Silin, Theory of a Degenerate Electron Liquid, Sov. Phys. JETP 6, 387 (1958).

[53] R. Lundgren, P. Laurell, and G. A. Fiete, Thermoelectric Properties of Weyl and Dirac Semimetals, Phys. Rev. B 90 , 165115 (2014).

[54] J. Xiang, S. Hu, Z. Song, M. Lv, J. Zhang, L. Zhao, W. Li, Z. Chen, S. Zhang, J. Wang, Y.-f. Yang, X. Dai, F. Steglich, G. Chen, and P. Sun, Giant Magnetic Quantum Oscillations in the Thermal Conductivity of TaAs: Indications of Chiral Zero Sound, arXiv:1801.08457.

[55] H. B. Nielsen and M. Ninomiya, Absence of Neutrinos on a Lattice: (II). Intuitive Topological Proof, Nucl. Phys. 193B, 173 (1981).

[56] H. B. Nielsen and M. Ninomiya, Absence of Neutrinos on a Lattice: (I). Proof by Homotopy Theory, Nucl. Phys. 185B, 20 (1981).

[57] A. A. Abrikosov, Quantum Magnetoresistance, Phys. Rev. B 58, 2788 (1998).

[58] P. Coleman, Introduction to Many-Body Physics (Cambridge University Press, Cambridge, England, 2015), Chap. 8, p. 267. 